\title{
Reputationsmanagement im Agribusiness
}

\author{
Dissertation \\ zur Erlangung des Doktorgrades \\ der Fakultät für Agrarwissenschaften \\ der Georg-August-Universität Göttingen
}

\author{
vorgelegt von \\ Friederike Albersmeier \\ geboren in Warburg
}

Göttingen, im Februar 2010 
D 7

1. Referent/Referentin:

Prof. Dr. Achim Spiller

2. Korreferent/Korreferentin:

Prof. Dr. Ludwig Theuvsen

Tag der mündlichen Prüfung: $\quad$ 26. Januar 2010 


\section{Inhaltsverzeichnis}

Einleitung. 1

Teil I:

Beziehung zwischen Agribusiness und Gesellschaft und die Bedeutung einer intakten Reputation

I.0 Einleitung: Zukunftsfaktor gesellschaftliche Akzeptanz: Kommunikation mit der

Öffentlichkeit - mehr als Krisen-PR

I.1 WAHRNeHMUNG UND BEDEUTUNG DER GESELlSCHAFT AUS Sicht DES

AGRIBUSINESS

I.1.1 Öffentlichkeitsorientierung in der Ernährungswirtschaft: Eine empirische Studie zum Umgang mit kritischen Anspruchsgruppen. 45

I.1.2 Zur Bedeutung gesellschaftlicher Anspruchsgruppen bei landwirtschaftlichen Bauprojekten: Einflussfaktoren auf einen erfolgreichen Stallbau 67

I.2 ZUR REPUTATION DER FLEISCHWIRTSCHAFT IN DER GESELLSCHAFT ..................... 78

I.2.1 Supply Chain Reputation in der Fleischwirtschaft............................... 78

I.2.2 Die Reputation der Fleischwirtschaft: Eine Kausalanalyse ................. 109

I.2.3 Das Ansehen der Fleischwirtschaft: Zur Bedeutung einer stufenübergreifenden Perspektive

I.2.4 Zur Wahrnehmung der Qualität von Schweinefleisch beim Kunden ... 169

\section{Teil II:}

Reputationsmanagement: Qualitätssicherung im Agribusiness

II.0 Einleitung: Risikoorientierte Prüfung in Zertifizierungssystemen der Land- und Ernährungswirtschaft

II.1 BEWERTUNG VON QUALITÄTSSICHERUNGSSYSTEMEN

II.1.1 The reliability of third-party certification in the food chain: From checklists to risk oriented auditing.

II.1.2 Heterogeneity in the Evaluation of Quality Assurance Systems: The International Food Standard (IFS) in European Agribusiness

II.1.3 Evaluation and reliability of the organic certification system: perceptions by farmers in Latin America 
II.2 ENTwickLUng EINEs MANAGEMENT-ToOLS ZUR ABSICHERUNG VOR REPUTATIONSSCHÄDEN.

II.2.1 System Dynamics in Quality Certifications: Development of an Audit Integrity System

II.2.2 The Application of Databases as an Information Management Tool in Quality Certification Systems

\section{Teil III:}

Exkurs: Beziehungsmanagement auf dem Landpachtmarkt

III.1 Die Bedeutung der Pachtpreisanpassungsklausel als Instrument des Risikomanagements

III.2 Die Bedeutung von Pachtpreisanpassungsklauseln auf dem Landpachtmarkt: Eine empirische Analyse von Verpächtern und Pächtern.

Resümee

Danksagung

Veröffentlichungs- und Vortragsverzeichnis

Lebenslauf. 


\section{Einleitung}

Die Agrar- und Ernährungswirtschaft hat in den zurückliegenden Jahrzehnten tiefgreifende Wandlungsprozesse durchlaufen. Die internen Veränderungen lassen sich insbesondere durch einen grundlegenden Strukturwandel beschreiben, in dessen Folge Spezialisierungs- und Konzentrationsgrade in den Nahrungsmittel-Wertschöpfungsketten angestiegen sind. Die Strukturen auf den einzelnen Stufen sowie die Organisation der gesamten Supply Chain haben sich nachhaltig gewandelt. Parallel zu den Veränderungen innerhalb der Branche vollzog sich auch ein Wandel in ihrem Umfeld. Hierbei sind insbesondere die Neuerungen im gesellschaftlichen Umfeld $\mathrm{zu}$ nennen. Die Gesellschaft hat sich heute weitgehend von der Landwirtschaft und der Lebensmittelverarbeitung entfremdet und nur noch geringe Teile der Bevölkerung verfügen über Wissen oder einen direkten Zugang zur Agrar- und Ernährungswirtschaft.

Die Wandlungsprozesse in der Agrarbranche und der Gesellschaft liefen dabei weitgehend getrennt voneinander ab, so dass sich beide Seiten zunehmend entfernt haben. Daraus resultieren heute zahlreiche Diskrepanzen zwischen den Erwartungen der Öffentlichkeit und der Wahrnehmung dieser Ansprüche durch die Branche. Dies haben nicht zuletzt auch die vielen Krisen und Skandale aufgezeigt. Insgesamt lassen sich heute tiefgreifende kulturelle Differenzen zwischen dem - größtenteils - eher städtischintellektuell geprägten Teil der Gesellschaft und der weitgehend ländlich-konservativen Agrarbranche konstatieren.

Diese Auseinandersetzungen zwischen Gesellschaft und Branche werden heute insbesondere durch die professionelle Kampagnenarbeit der Nicht-Regierungs-Organisationen (NGO) weiter vorangetrieben. Beispiele für derartige „Kulturkonflikte“ sind u. a. der Ökolandbau, Tierschutz, der Einsatz der Grünen Gentechnik oder die Klimarelevanz des Agrarsektors. Ihren Anfang nahmen diese offen ausgetragenen Konflikte in den 1990er Jahren mit dem BSE-Skandal und setzten sich über die Schweinepest und umetikettierte Fleischwaren im Gammelfleischskandal fort. Dabei war das gesellschaftliche Augenmerk zunächst vornehmlich auf den Primärsektor gerichtet. Heute stehen indessen vermehrt auch die nachgelagerten Stufen im Blickfeld der Gesellschaft. Neben der Erweiterung des öffentlichen Aufmerksamkeitsradius veränderten sich auch die Themenfelder, mit denen sich die Branche konfrontiert sieht. Dabei sind es nicht allein die Produktqualität und die Warensicherheit, die einer gesteigerten öffentlichen 
Beobachtung unterliegen, sondern insbesondere soziale, ethische und ökologische Aspekte der Produktion, die oftmals in die Kritik geraten. Während auf der landwirtschaftlichen Stufe vor allem Prozessqualitäten wie Tier- und Umweltschutz angesprochen werden, rücken auf den nachfolgenden Stufen besonders moralische (Verbrauchertäuschung und -betrug u. a. im Gammelfleischskandal) und soziale Aspekte (schlechte Arbeitsverhältnisse für die zumeist ausländischen Mitarbeiter) in das Blickfeld der Öffentlichkeit. Insbesondere der Fleischsektor und seine Erzeugnisse haben in der Gesellschaft zunehmend an Vertrauen und Glaubwürdigkeit verloren und werden infolgedessen zum Ziel von Kampagnen kritischer Anspruchsgruppen.

Die Beziehung zwischen Agrarsektor und Öffentlichkeit bzw. Medien ist durch diese Vorfälle insgesamt schwer beschädigt und von großem Misstrauen geprägt. Dieses Misstrauen belastet zunehmend auch die eigentlich zum Vertrauensaufbau und zur Reduktion von Informationsasymmetrien implementierten Maßnahmen, wie bspw. Zertifizierungssysteme. In der jüngeren Vergangenheit kam es in diesem Zusammenhang immer wieder zu kritischen Äußerungen, Angriffen und konkreten Kampagnen von verschiedenen Initiativen und Organisationen (vor allem aus den Bereichen Tier-, Umwelt- und Verbraucherschutz) gegen einige der etablierten Standards. Insbesondere die Funktionsfähigkeit und Glaubwürdigkeit des privatwirtschaftlich organisierten QSSystems (Qualität und Sicherheit) wurde von Nichtregierungsorganisationen wie Foodwatch oder PETA (People for the Ethical Treatment of Animals) zunehmend öffentlich infrage gestellt. ${ }^{1}$ Da jeder Standard seine Legitimation und damit seinen Erfolg aus dem ihm entgegengebrachten Vertrauen zieht, stellen derartige Kampagnen eine nicht zu unterschätzende Bedrohung für die Standards und auch für die gesamte Branche dar, weil sie das Verbrauchervertrauen erodieren.

Die vorliegende kumulative Dissertation greift die skizzierten Problemstellungen aus theoretischer und empirischer Perspektive - vorrangig am Beispiel der Fleischwirtschaft - auf. Der Fokus der Arbeit liegt dabei auf der öffentlichen Reputation der Branche und der Qualitätssicherung von Lebensmitteln als ein Instrument des proaktiven Reputations-Managements. Insgesamt umfasst die Arbeit 15 bereits publizierte oder eingereichte Beiträge. Die Studien sind drei inhaltlichen Schwerpunkten zugeordnet -

\footnotetext{
${ }^{1}$ Beispiele für Kampagnen von Foodwatch und PETA unter http://www.foodwatch.de/kampagnen themen/qualitaetssiegel/index_ger.html oder www.peta.de/qs2009). QS reagierte auf die PETA-Angriffe mit einer gerichtlichen Klage (http://www.q-s.de/de/mediacenter/news/artikel/qs-vor-dem-landgerichthamburg-erfolgreich-1/).
} 
neben den beiden aufgezeigten Themenkomplexen „Zur Beziehung von Agribusiness und Gesellschaft“ und „Qualitätssicherung im Agribusiness“ werden in einem Exkurs weitere Beiträge präsentiert, die nicht in einem direkten thematischen Kontext zum Promotionstitel stehen. Abbildung 1 stellt den generellen Aufbau der Arbeit dar.

Abbildung 1: Aufbau der Arbeit

Einleitung

\begin{tabular}{|c|c|c|}
\hline \multicolumn{3}{|c|}{ Einleitung } \\
\hline & $\begin{array}{c}\text { Teil I Beziehung zwischen Agribusiness und Gesellschaft und die } \\
\text { Bedeutung einer intakten Reputation }\end{array}$ & \multirow{8}{*}{ 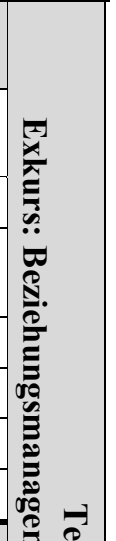 } \\
\hline & $\begin{array}{l}\text { I.0 Einleitung Zukunftsfaktor gesellschaftliche Akzeptanz: Kommunikation } \\
\text { mit der Öffentlichkeit - mehr als Krisen-PR }\end{array}$ & \\
\hline \multirow{6}{*}{$\begin{array}{l}\text { Problemstellung } \\
\text { (betrachtet aus } \\
\text { verschiedenen } \\
\text { Perspektiven) }\end{array}$} & I.1.1 Zur Öffentlichkeitsorientierung der Ernährungswirtschaft & \\
\hline & $\begin{array}{l}\text { I.1.2 Wahrnehmung und Stellenwert gesellschaftlicher Ansprüche durch die } \\
\text { Landwirtschaft }\end{array}$ & \\
\hline & I.2.1 Supply Chain Reputation in der Fleischwirtschaft & \\
\hline & I.2.2 Die Reputation der Fleischwirtschaft in der Gesellschaft & \\
\hline & I.2.3 Zur Branchen-Reputation aus einer stufenübergreifenden Perspektive & \\
\hline & I.2.4 Zur Wahrnehmung der Qualität von Schweinefleisch beim Kunden & \\
\hline \multirow{2}{*}{$\begin{array}{l}\text { Maßnahmen (zur } \\
\text { Verbesserung der } \\
\text { Beziehung) }\end{array}$} & Teil II Reputations-Management: Qualitätssicherung im Agribusiness & \multirow{6}{*}{ 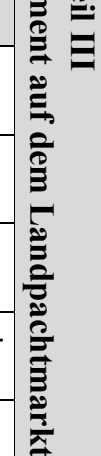 } \\
\hline & $\begin{array}{l}\text { II.0 Einleitung Zertifizierungssysteme in der Land- und } \\
\text { Ernährungswirtschaft }\end{array}$ & \\
\hline \multirow{3}{*}{$\begin{array}{l}\text { Bewertung der } \\
\text { Systeme }\end{array}$} & $\begin{array}{l}\text { II.1.1 Evaluation der Glaubwürdigkeit von Zertifizierungssystemen (QS) } \\
\text { auf Basis von Auditdaten }\end{array}$ & \\
\hline & $\begin{array}{l}\text { II.1.2 Bewertung von QAS (IFS) aus Nutzersicht - Unternehmen der } \\
\text { Ernährungsindustrie }\end{array}$ & \\
\hline & $\begin{array}{l}\text { II.1.3 Bewertung von QAS (Öko-Zertifizierung) aus Nutzersicht- Land- } \\
\text { wirte in Lateinamerika }\end{array}$ & \\
\hline \multirow{2}{*}{$\begin{array}{l}\text { Maßnahmen (zur } \\
\text { Verbesserung der } \\
\text { Systeme) } \\
\end{array}$} & II.2.1 System Dynamics: Development of an Audit Integrity System & \\
\hline & II.2.2 The Application of Databases in Quality Certification Systems & \\
\hline Ziel & Absicherung der Reputation & \\
\hline
\end{tabular}

Nachdem zuvor bereits eine kurze Einleitung in die Problemstellung der Arbeit gegeben wurde, werden im Nachfolgenden die Inhalte der beiden Hauptteile spezifischer und nacheinander vorgestellt. Anschließend erfolgt eine knappe Beschreibung sämtlicher 15 Beiträge in Form ihrer jeweiligen Zusammenfassungen. 


\section{Beziehung zwischen Agribusiness und Gesellschaft und die Bedeutung einer intakten Reputation}

Im ersten Teil der vorliegenden Arbeit werden sechs Beiträge vorgestellt, die sich schwerpunktmäßig mit der Beziehung zwischen der Gesellschaft sowie dem Agribusiness und seinen Unternehmen auseinander setzen. Der erste Beitrag ist eine Einführung in den Themenkomplex I und gibt einen kurzen Überblick über die Bedeutung der Gesellschaft als Anspruchsgruppe der Agrar- und Ernährungsindustrie und beschreibt ferner den Umgang der Branche mit öffentlichen Ansprüchen und Forderungen. Erste Ansätze eines professionellen PR-Managements für das Agribusiness werden aufgezeigt. An den Beispielen der Ernährungsindustrie und der Landwirtschaft wird die Öffentlichkeitsorientierung des Agrarsektors dann nachfolgend konkretisiert (vgl. I.1). Darüber hinaus ist die Untersuchung der Branchen-Reputation in der Gesellschaft ein maßgeblicher Forschungsschwerpunkt der präsentierten Dissertation. Anhand der Fleischwirtschaft werden dabei das grundsätzliche Reputationsniveau des Sektors aufgezeigt und die wesentlichen Einfluss- und Erklärungsgrößen auf den Ruf identifiziert (vgl. I.2).

Für viele Unternehmen der Agrar- und Ernährungswirtschaft ist der Umgang mit einer mündigen Öffentlichkeit und ihren professionalisierten Protestorganisationen ein schwieriges Thema. Entfremdungsprozesse in der Gesellschaft haben dazu geführt, dass die Öffentlichkeit heute weitgehend keine Kenntnisse mehr über den Agrarbereich besitzt. Oftmals ist auch das Wissen der Branche um die Funktionsweise von Medien, Meinungsmachern und Kampagnenarbeit eher gering. Viele Unternehmen verhalten sich hier schlichtweg naiv und haben es nicht verstanden, gesellschaftliche Ansprüche an ihr Unternehmen bzw. ihren landwirtschaftlichen Betrieb ernst zu nehmen (Albersmeier et al., 2008; Schlecht et al., 2008).

Dies schlägt sich in einer schlechten Reputation der Branche wieder, d. h. einer negativen Evaluation der Leistungen und Eigenschaften der Branche. Die negativen Auswirkungen einer schlechten Reputation bzw. die Potentiale eines intakten Ansehens werden von den Betrieben im Agribusiness weitgehend verkannt. Dabei konnte bereits in zahlreichen Studien die Ausbildung vielfältiger Unterstützungspotentiale bei marktlichen und nicht-marktlichen Anspruchsgruppen sowie die Aufrechterhaltung einer intakten Reputation im Krisenfall nachgewiesen werden (Mast et al., 2005; 
Wiedmann et al., 2006). Denn Unternehmen mit einer hohen Reputation wird ein hohes $\mathrm{Ma} ß$ an Vertrauen entgegengebracht - ihnen wird funktionsgerechtes sowie normativ und moralisch korrektes Handeln unterstellt.

Auf Seiten der Kunden/Verbraucher sind insbesondere die Kauf-, Preis- und Weiterempfehlungsbereitschaft für Unternehmen mit einer guten Reputation wesentlich stärker ausgeprägt als bei anderen. Das heißt, Konsumenten kaufen eher bei Firmen, die sich sozial engagieren, sind potentiell eher bereit, einen höheren Preis für Produkte dieser Unternehmen zu zahlen, und bleiben diesen auch nachhaltig treu (Smith, 2003; Du et al., 2007; Eccles et al., 2007). Gleiches gilt für Lieferanten und die Wirkung der Firma auf den Arbeitsmarkt (Aron, 1987). Smith (2003: 63) zeigt auf, dass Arbeitnehmer klare Präferenzen für sozial verantwortliche Unternehmen mit einer guten öffentlichen Reputation aufweisen. Insbesondere auf eng umkämpften Arbeitsmärkten, bei denen mehrere Firmen um potentielle Mitarbeiter werben, selektiert der Arbeitnehmer auch hinsichtlich des Rufes von Unternehmen. Im Extremfall verzichten Mitarbeiter auf Gehalt und andere Incentives, um in einem Unternehmen mit einer intakten Reputation $\mathrm{zu}$ arbeiten. Auch beim Halten von alten Mitarbeitern ist die Reputation von Bedeutung (Mahon, 2002). Letztere zeichnen sich in Firmen mit einem guten Ansehen allgemein durch eine hohe Moral und Produktivität aus (Gray und Balmer, 1998). Dass die Reputation auch für die Investoren auf dem Kapitalmarkt von nicht zu vernachlässigender Bedeutung ist, zeigt die Einführung von diversen Indizes ${ }^{2}$, die Unternehmen im Hinblick auf das Erreichen von verschiedenen wirtschaftlichen, sozialen und ökologischen Kriterien bewerten (Smith, 2003; Mahon, 2002). Diese Nachhaltigkeitsbewertung soll dem Anleger als Entscheidungshilfe bei seinem Investitionsverhalten dienen. Auf Seiten der Unternehmen hat diese Entwicklung dazu geführt, dass eine steigende Zahl an Unternehmen eigene Nachhaltigkeitsberichte erstellt und als Instrument zum Reputationsaufbau auf dem Kapitalmarkt nutzt.

Im Hinblick auf die Öffentlichkeit - und im Speziellen NGOs oder Bürgerinitiativen generiert ein hohes Ansehen eine verstärkte Einflussnahme auf den Verlauf gesellschaftlicher Themen (Gray und Balmer, 1998; Mast et al., 2005). Dies ist insbesondere im politischen Diskurs von entscheidender Bedeutung. Das Maß, in dem eine ganze Branche im Politikfeld beachtet und zum Gegenstand von Debatten wird, ist immer

\footnotetext{
2 z. B. Dow Jones Sustainability Index, Domini 400 Social Index, Ethical Index Global, Natur-AktienIndex (NAI), FTSE4Good
} 
auch abhängig von dem Ruf der jeweiligen Industrie. Auf der Verwaltungsebene spiegelt sich eine negative Reputation der Branche vor allem in einem erhöhten staatlichen Kontrolldruck wider (Gray und Balmer, 1998).

Wer somit über genug Anerkennung bei den aufgezeigten Gruppen verfügt, wird eher über nachhaltige Unterstützung verfügen, um die verschiedenen Unternehmensziele langfristig zu erreichen (ibid.). Dabei kommen die Reputation bzw. die daran geknüpften Unterstützungspotentiale und der Vertrauensvorschuss insbesondere in Krisenzeiten zum Tragen: Schwalbach (2002) zeigte in einer Studie, dass die Reputation zwar grundsätzlichen Schwankungen unterliegt, Unternehmen oder Branchen mit einer positiven Reputation jedoch deutlich beständiger und weniger krisenanfällig sind. Die Reputation vor dem Krisenfall hat somit entscheidenden Einfluss auf die Überwindung der selbigen (Davies et al., 2003; Fombrun und van Riel, 2003). Coombs und Holladay (2006: 123) vergleichen die Reputation mit einem Bankkonto, welches in schlechten Zeiten belastet wird: Bei einer hohen, stabilen Reputation sind die Verluste weniger bedeutend als bei einem strapazierten Konto.

Insbesondere in einer auf messbare, quantitative Erfolgsgrößen ausgerichteten Branche wie der Fleischwirtschaft ist es schwer, die zuvor beschriebenen Potentiale einer intakten Reputation zu kommunizieren und entsprechende Ressourcen für PR- und Kommunikationsabteilungen abzustellen. Denn oftmals lassen sich die aufgezeigten Unterstützungspotentiale nur schwer eindeutig zuordnen und von anderen Effekten isolieren. Infolgedessen können sie auf betrieblicher Ebene fast nicht gemessen und quantifiziert werden. In verschiedenen wissenschaftlichen Untersuchungen konnte dagegen ein positiver Zusammenhang zwischen „Reputation“ und „finanziellem Erfolg“ bestätigt werden (Fuentes Sabat und Quevedo Puente, 2003; Abraham et al., 2005). Die betrachteten Arbeiten zeigen dabei sowohl den Einfluss, den der finanzielle Erfolg auf die organisationale Reputation ${ }^{3}$ besitzt, als auch den umgekehrten Fall ${ }^{4}$ und eine generelle Interaktion zwischen beiden Strukturen ${ }^{5}$ (Fuentes Sabat und Quevedo Puente, 2003; Herger, 2006).

\footnotetext{
${ }^{3}$ vgl. Sobol und Farelly, 1988; Fombrun und Shanley, 1990; Riahi-Belkaoui und Pavlik, 1991; Hammond und Slocum, 1996

${ }^{4}$ vgl. Srivastava et al., 1997; Deephouse, 1997; Roberts und Dowling, 1997; Cordeiro und Sambharya, 1997; Vergin und Qoronfleh, 1999; Jones et al., 2000

${ }^{5}$ vgl. McGuire, 1990; Dunbar und Schwalbach, 2000; Chung et al., 1999
} 
Dass die Wahrnehmung und der Stellenwert in der Gesellschaft für das Agribusiness gering sind und in vielen der meist mittelständischen Unternehmen der Agrarbranche, aber auch in den großen Schlacht- und Verarbeitungsbetrieben der Fleischwirtschaft, entsprechende Organisationsstrukturen fehlen, die die Reputation der Unternehmen und somit indirekt auch der Branche - steuern können, wird insbesondere im ersten Teil der Dissertation deutlich (vgl. I.1). Der erste Abschnitt I.1 gliedert sich dabei in zwei Beiträge: Während die eine Studie die grundsätzliche Öffentlichkeitsorientierung von Vertretern der Ernährungswirtschaft mittels einer empirischen Befragung erhebt, fokussiert die zweite Studie auf die Stufe der Primärproduktion. Dieser Beitrag untersucht anschaulich am Beispiel des Stallbaus die vielfältigen gesellschaftlichen Akzeptanzprobleme, mit denen sich die Landwirtschaft heute auseinandersetzen muss. Aus Sicht der Landwirte (mit und ohne Konflikterfahrung beim Bau von Schweineställen) wird hierbei insbesondere die Bedeutung der Gesellschaft für die Landwirtschaft sowie konkret bei der Realisierung von Stallbauprojekten eruiert.

In Abschnitt I.2 erfolgt dann ein Perspektivwechsel - in vier Beiträgen wird hier untersucht, wie das Produkt Fleisch und die Fleischwirtschaft von der Gesellschaft wahrgenommen und bewertet wird. Zur Messung der Akzeptanz greifen diese Arbeiten maßgeblich auf die Theorie der Reputationsforschung zurück. Der erste Beitrag stellt das Konstrukt der Reputation vor und gibt einen Überblick über die verschiedenen Messinstrumente. Während die etablierte Literatur vorrangig auf die Reputation von Unternehmen abzielt, setzt sich dieser Beitrag in konzeptionell-theoretischer Weise mit dem Ansehen von ganzen Branchen auseinander. Bei der zweiten Studie handelt es sich um eine empirische Untersuchung, die auf Basis einer Kausalanalyse (unter Verwendung des „Partial Least Square“ (PLS) Verfahrens) Einflussgrößen auf den Ruf der gesamten Branche sowie Auswirkungen der schlechten Reputation auf verschiedene gesellschaftliche Akzeptanzfaktoren ermittelt. Der dritte Beitrag greift die gewonnenen Ergebnisse auf und analysiert das Ansehen der Fleischwirtschaft aus einer Supply Chain Perspektive. Hierbei stehen insbesondere die Zusammenhänge zwischen dem Ruf der einzelnen Wertschöpfungsstufen sowie Auswirkungen der jeweiligen Stufenreputation auf gesellschaftliche Akzeptanzgrößen der vor- und nachgelagerten Kettenmitglieder im Mittelpunkt der Untersuchung. Im Fokus des vierten Beitrags steht nicht die Fleischbranche selbst, sondern die Bewertung der erzeugten Produkte durch die Konsumenten. Insbesondere die Wahrnehmung der Fleischqualität und das Wissen um Fleisch beim 
Einkauf soll hierbei erhoben und analysiert werden. Mittels eines Wahlexperiments wurde die Präferenz für unterschiedlich marmoriertes und gefärbtes Fleisch, welches darüber hinaus ferner im Preis und der Applikation eines Qualitätssiegels divergierte, untersucht. Auch hier zeigen sich deutliche Kommunikations- und Informationsdefizite.

\section{Reputationsmanagement: Qualitätssicherung im Agribusiness}

Im zweiten Themenkomplex der Arbeit werden fünf Beiträge präsentiert, deren Fokus auf der Untersuchung der unterschiedlichen Qualitätssicherungsstandards in der Landund Ernährungswirtschaft liegt. Die Hintergründe für die Entwicklung derartiger Systeme sowie mögliche Risiken und Probleme, mit denen sie konfrontiert sind, werden in einer Einleitung in diesem Teil der Dissertation dargestellt. Neben einer grundsätzlichen Evaluation und Bewertung der Glaubwürdigkeit dieser Systeme (vgl. II.1) liegt ein besonderer Schwerpunkt der Arbeit auf den Potentialen der Entwicklung von Management-Tools, die die stetig wachsenden Standards vor möglichen Reputationsschäden schützen sollen (vgl. II.2).

Die Entstehung der verschiedenen Qualitätssicherungssysteme in der europäischen Land- und Agrarwirtschaft begründet sich in den zahlreichen Lebensmittelkrisen und -skandalen, die Anfang der 90iger Jahre mit BSE ihren Anfang nahmen (Tuncer, 2001). Diese Ereignisse haben die Mechanismen und Systeme zum Management von Lebensmittelqualität und -sicherheit in ganz Europa stark beeinflusst und grundlegend verändert. Triebkräfte für diese Entwicklung war insbesondere der Handel, der hiermit nicht zuletzt auf das infolge der Krisen gesteigerte Interesse und die hohe Nachfrage der Konsumenten nach Informationen über die Produktion sowie Qualität von Nahrungsmitteln reagierte (Hatanaka et al., 2005; Fulponi, 2006). Das erschütterte Vertrauen der Gesellschaft in die Funktionsfähigkeit von staatlichen Institutionen und Kontrollstellen sowie in das Agribusiness insgesamt (Pennings et al., 2002; Poppe und Kjaernes, 2003; Albersmeier und Spiller, 2009), spielte dabei eine maßgebliche Rolle für die Implementierung von neutralen Überwachungssystemen.

Eine Ursache für das entstandene Mistrauen und die Verbraucherverunsicherung war vor allem die weitgehend ungleiche Verteilung von Informationen zwischen Produzenten und Verbrauchern im Hinblick auf die Erzeugung und Verarbeitung von Produkten (Marten, 1999). Dies gilt insbesondere für die Agrar- und Ernährungsindustrie, da hier Anbau- und Verarbeitungsmethoden vergleichsweise komplex und die Prozessattribute 
oft nicht mehr rückwirkend nachweisbar sind (Caswell und Mojduszka, 1996; Luning und Marcelis, 2005). Lebensmittel verfügen über eine Vielzahl an Vertrauens- bzw. Potemkin-Eigenschaften (Bodenstein und Spiller, 1998), die sich dadurch auszeichnen, dass es den Nachfragern entweder nur mit sehr hohem Aufwand (Analyse des Endproduktes auf z. B. Strahlen- oder Schadstoffbelastung durch Testinstitute oder andere Institutionen) oder auf keinerlei Weise möglich ist (z. B. artgerechte Tierhaltung oder ökologischer Anbau), entsprechende Prozessqualitäten von Lebensmitteln zu überprüfen (Antle, 2001; Jahn et al., 2005). Ziel der diversen Zertifizierungssysteme ist es daher, die Produktions- und Verarbeitungsprozesse in der Agri-Food Chain zu überwachen und die Informationslücke zu schließen (Auril und Schilizzi, 2002). Durch entsprechende Label sollen den Verbrauchern die entsprechenden Prozesseigenschaften der Produkte signalisiert werden.

Große Teile der Nahrungsmittelkette sind daher heute nach privatwirtschaftlichen Standards wie QS (Qualität und Sicherheit GmbH), IFS (International Food Standard) oder GLOBALGAP (Global Partnership for Good Agricultural Practice) zertifiziert. Diese unterschieden sich in ihrer spezifischen Ausprägung und Zielsetzung, ihr Aufbau bzw. die institutionelle Struktur ist jedoch für die verschiedenen Standards grundsätzlich vergleichbar (Theuvsen und Spiller, 2007). Die weltweit etablierten Standards stellen jedoch ihrerseits wiederum für den Konsumenten ein Vertrauensgut dar, da die an die Systemteilnehmer gestellten Anforderungen und die Qualität der Kontrollsysteme für Dritte nur begrenzt beurteil- und bewertbar sind. Für Standards ist es daher entscheidend, die Vertrauenswürdigkeit des Systems glaubwürdig zu kommunizieren und eine positive Reputation aufzubauen (Golan et al., 2001). Um ihre Existenzberechtigung zu untermauern, ist es für die Standards entscheidend, Schwachstellen im System aufzudecken und die Qualität der Prüfung zu steigern. Ohne eine glaubwürdige und verlässliche Prüfung sind Zertifikate nur „Potemkinsche Dörfer im Dschungel der Bürokratie" (Schulze et al., 2006: 4) und werden langfristig an Akzeptanz verlieren. Es stellt sich somit die Frage, ob die Qualitätssicherungssysteme de facto ihre gesteckten Ziele erreichen können und eine hinreichende Kontrollvalidität und -reliabilität garantieren.

Neben Schwachstellen in der Ausgestaltung des Standards und seines Kontrollprozesses selbst, sind es jedoch auch die Systemteilnehmer, die die Validität und Reliabilität des Systems ins Wanken bringen können. Die Zufriedenheit der verschiedenen Destinatare 
innerhalb des Qualitätssicherungsstandards (z. B. Landwirte oder verarbeitende Unternehmen) ist dabei ein wesentliches Kriterium für ihren Erfolg und die Stabilität (Böcker et al., 2003; Jahn und Spiller, 2005). Hierdurch können Akzeptanz und eine positive Motivation bei den Nutzern generiert werden. Nur Standards, die den intrinsischen Antrieb von Anwendern fördern und die nicht als „bürokratische Last“ wahrgenommen werden, werden langfristig zu Qualitätsverbesserungen im gesamten System führen.

Vor diesem Hintergrund stehen bei den drei in Abschnitt II.1 vorgestellten Beiträgen die Evaluation der Funktionsfähigkeit des Kontrollprozesses sowie die Glaubwürdigkeit des gesamten Systems im Vordergrund. In Beitrag II.1.1 wird auf Basis einer Auswertung der Prüfdatenbank des deutschen QS-Systems untersucht, ob die neutrale Kontrolle des Standards tatsächlich als reliabel beurteilt werden kann. Ziel der Studie ist es, erste empirische Hypothesen zum Zusammenhang zwischen Auditqualität und institutionellem Rahmen der Zertifizierung abzuleiten. Darauf aufbauend erfolgt eine Vorstellung des risikoorientierten Prüfungsansatzes aus der Wirtschaftsprüfung. Es wird diskutiert, inwieweit dieses Konzept ein geeigneter Ausgangspunkt sein kann, um die Effizienz und Effektivität von Kontrollen in Zertifizierungssystemen der Land- und Ernährungswirtschaft zu verbessern. Im zweiten und dritten Artikel werden die relevanten Determinanten der Einstellungen und Evaluation von Nutzern im Hinblick auf Qualitätssicherungsstandards aus einer verhaltenswissenschaftlichen Perspektive identifiziert. Die zweite Studie untersucht dabei anhand einer umfangreichen empirischen Befragung die Bewertung des International Food Standards (IFS) durch Unternehmen der europäischen Agri-Food Chain. Der Fokus der Studie liegt auf der Identifikation von verschiedenen Gruppen innerhalb des Samples, die hinsichtlich der Beurteilung des IFS divergieren. Im dritten Beitrag wird durch die Anwendung der PLS-Methode ein Strukturgleichungsmodell berechnet, welches die Einfluss- und Erklärungsgrößen auf die Bewertung des Öko-Zertifizierungssystems bei Landwirten in Lateinamerika aufdecken soll. Hierbei stand der Zusammenhang zwischen der Evaluation des Systems und der wahrgenommenen Glaubwürdigkeit im Mittelpunkt der Analysen.

Aufbauend auf diesen Arbeiten, die die Glaubwürdigkeit verschiedener Systeme aus unterschiedlichen Perspektiven untersucht haben, fokussiert Abschnitt II.2 die Entwicklung eines anwendungsorientierten Management-Tools zur Identifikation und Regulation von Schwachstellen in den Systemen. In den beiden nachstehenden, konzeptionellen Studien wird, aufbauend auf dem risikoorientierten Prüfungsansatz, das „Audit 
Integrity System“ vorgestellt. Durch dieses wird es den Standardeignern von Zertifizierungssystemen möglich, auf Basis einer automatisierten, EDV-gestützten Auswertung ihrer Datenbanken kontinuierlich die (Prüf-) Qualität ihres Standards zu optimieren. Das Hauptziel eines derartigen Tools ist der Schutz vor Reputationsschäden, die das gesamte System zum Kollabieren bringen können. Denn nur funktionsfähige Standards genießen das Vertrauen der Verbraucher sowie der Akteure des Agribusiness und besitzen somit eine beständige und strapazierfähige Legitimationsgrundlage.

\section{Exkurs}

Der letzte Teil der Arbeit fasst zwei Studien zusammen, die im Rahmen der Promotion angefertigt wurden, aber nicht im engeren thematischen Kontext mit dem Titel der Dissertation stehen. Bei den Beiträgen handelt es sich um zur Publikation eingereichte Studien, die sich mit den Herausforderungen auf dem Landpachtmarkt beschäftigen und hierbei insbesondere der Frage nachgehen, wie ein Pachtverhältnis langfristig die Interessen von Pächtern und Verpächtern ausgewogen berücksichtigen kann.

Die Pacht von landwirtschaftlichen Flächen nimmt in Deutschland eine herausragende Stellung ein. In den vergangenen Jahren konnte ein Anstieg des durchschnittlichen Pachtflächenanteils von 53,3 \% im Jahr 1991 auf 61,8 \% pro Betrieb im Jahr 2007 ermittelt werden (Destatis, 1992, 2008). Aufgrund der Bedeutung des Bodens als wichtigster Produktionsfaktor und maßgebliche Kostenstelle in landwirtschaftlichen Betrieben nehmen die Entwicklungen auf dem Pachtmarkt starken Einfluss auf deren strategische Entwicklungsmöglichkeiten (Chaztis, 1996; Theuvsen, 2007; DBV, 2009). Vor allem die Realisierung von Wachstumsstrategien und die Investitionsbereitschaft von Landwirten werden hierdurch maßgeblich beeinflusst (Doll, 2002; Bertelsmeier, 2004; Plumeyer, 2006).

In den beiden Beiträgen des Kapitels III werden daher neben der Analyse der Relevanz und Verbreitung von Pachtpreisanpassungsklauseln (PPAK) bei Pächtern und Verpächtern insbesondere auch die Einflussgrößen auf deren Nutzung untersucht. Die Einflussstärke der verschiedenen Parameter wurde dabei für die unterschiedlichen Nutzer-Gruppen von PPAK (Nutzer, Interessierte und Ablehner) anhand einer multinomialen logistischen Regression analysiert. Während die erste Studie die relevanten Faktoren für die Anwendung von PPAK in Pachtverträgen für die Landwirte identifiziert, wird im zweiten Beitrag ein Vergleich der Einflussgrößen zwischen Päch- 
tern und Verpächtern durchgeführt. Ziel der beiden Arbeiten ist es hierbei, die wesentlichen Adaptionsbarrieren $\mathrm{zu}$ identifizieren und wichtige Ansatzpunkte zur Förderung der Verbreitung von PPAK bei Pächtern und Verpächtern zu geben.

Nachfolgend werden alle Beiträge der Dissertation in Form von kurzen Abstracts vorgestellt und im Anschluss in der publizierten Version präsentiert. Die Arbeit wird durch eine Liste mit sämtlichen wissenschaftlichen und praxisorientierten Publikationen und Vorträgen ergänzt, die während der Promotionszeit entstanden sind bzw. gehalten wurden. 


\section{Teil I: Beziehung zwischen Agribusiness und Gesellschaft und die Bedeutung einer intakten Reputation}

\section{I.0 Einleitung: Zukunftsfaktor gesellschaftliche Akzeptanz: Kom- munikation mit der Öffentlichkeit - mehr als Krisen-PR}

Autoren: Justus Böhm, Friederike Albersmeier, Achim Spiller und Anke Zühlsdorf

Erschienen in: Böhm, J., Albersmeier, F., Spiller, A. (Hrsg.): Die Ernährungswirtschaft im Scheinwerferlicht der Öffentlichkeit, Eul-Verlag, 3-22, (2009). ${ }^{6}$

Der Umgang mit der kritischen Öffentlichkeit ist für viele Unternehmen der Agrar- und Ernährungswirtschaft ein heikles Thema. Das Verhältnis zu Öffentlichkeit und Medien ist für das Gros der Branche schwierig und seit Jahren belastet. Skandale und Krisen wie BSE, Nitrofen oder Gammelfleisch haben den Ruf der Ernährungswirtschaft empfindlich geschädigt und den Scheinwerfer des gesellschaftlichen Interesses immer stärker auf die Nahrungsmittelproduktion - und speziell die Fleischwirtschaft - gelenkt. Während die öffentliche Exponiertheit für die Akteure insgesamt gewachsen ist, setzte die Agrarbranche bislang auf eine Abtauch- bzw. Low Profile-Strategie, um öffentliche Aufmerksamkeit bewusst zu meiden. Handlungsstrategien für einen konstruktiven Umgang mit der Öffentlichkeit sind (noch) unterentwickelt. Langfristig ist ein Low Profile als sehr kritisch zu betrachten, da Krisenfälle Reputationsschäden und einen Verlust an Vertrauen bei Verbrauchern, Politikern und Medien nachsichziehen. Der Verlust an Ansehen kann die zukünftigen Handlungsoptionen eines Unternehmens erheblich einschränken. Insbesondere in Krisenzeiten wirkt ein guter Ruf wie ein Schutzschild, an dem Angriffe von Kritikern abprallen. Bei schlechter Reputation werden Aussagen eines Unternehmens dagegen permanent hinterfragt. Entscheidend für die Agrarbranche sind daher die präventiven Kommunikationsmaßnahmen vor der Krise: Hier geht es um Zukunftsinvestitionen in die Beziehungsqualität - zur allgemeinen Öffentlichkeit, zu Journalisten und auch zu kritischen Anspruchsgruppen.

\footnotetext{
${ }^{6}$ Der Beitrag wurde in ähnlicher Fassung veröffentlicht: Albersmeier, F., Böhm, J., Spiller, A., Schattke, H., Pfriem, R. (2009): Die Fleischwirtschaft im Scheinwerferlicht der Öffentlichkeit: Zukunftsfaktor gesellschaftliche Akzeptanz. In: Die Fleischwirtschaft (9), 71-77.
} 


\section{I.1 WAHRNehMUNG UND BEDEUTUNG DER GeSEllSChAFT AUS SiCHT DES AGRIBUSINESS}

\section{I.1.1 Öffentlichkeitsorientierung in der Ernährungswirtschaft: Eine empirische Studie zum Umgang mit kritischen Anspruchs- gruppen}

Autoren: Friederike Albersmeier, Achim Spiller und Katrin Jäckel

Erschienen in: Zeitschrift für Management 3 (4), 363-383, (2008).

Die gesellschaftliche Verantwortung von Unternehmen ist in der Managementlehre seit langem Forschungsgegenstand. Der vorliegende Beitrag setzt sich empirisch mit dem Themenfeld auseinander. Exemplarisch wird dafür die deutsche Ernährungswirtschaft herangezogen, die bislang nur über begrenzte Erfahrungen mit kritischen Stakeholdern verfügt. Ziel ist es, den Grad der Sensitivität von Unternehmen gegenüber Ansprüchen der Gesellschaft zu untersuchen. Die Studienergebnisse zeigen eine eher gering ausgeprägte Öffentlichkeitsorientierung des Agribusiness. Aufgrund der zunehmenden Relevanz gesellschaftlicher Stakeholder wird eine Professionalisierung der PR-Arbeit jedoch immer relevanter. Das im Weiteren entwickelte Konzept der Öffentlichkeitsorientierung bietet hierfür hilfreiche Ansatzpunkte. 


\section{I.1.2 Zur Bedeutung gesellschaftlicher Anspruchsgruppen bei land- wirtschaftlichen Stallbauprojekten: Einflussfaktoren auf einen erfolgreichen Stallbau}

Autoren: Friederike Albersmeier, Stephanie Schlecht und Achim Spiller

Erschienen in: ÖGA-Jahrbuch 18 (1), 1-10, (2009). ${ }^{7}$

In den letzten Jahren werden landwirtschaftliche Stallbauprojekte zunehmend von Konflikten mit der lokalen Öffentlichkeit begleitet. Bisher liegen wenige empirische Studien zu diesem Phänomen vor. Der folgende Beitrag analysiert, welche Bedeutung gesellschaftlichen Stakeholdern bei Stallbauvorhaben zukommt, und welche Faktoren das Auftreten von Auseinandersetzungen bestimmen. Eine Logit-Regression illustriert, dass insbesondere die Stellung der Landwirtschaft im eigenen Ort, deren Image sowie die Lage des Betriebes die Eintrittswahrscheinlichkeit von Stallbaukonflikten beeinflussen. Auf Grundlage dieser Ergebnisse können sich Landwirte über geeignete Strategien informieren und aktiv auf den Erfolg ihres Bauprojekts einwirken.

\footnotetext{
${ }^{7}$ Der Beitrag wurde in ähnlicher Fassung veröffentlicht: Spiller, A.; Albersmeier, F.; Schlecht, S. (2009): Nachbarschaftsärger beim Stallbau vermeiden. In: Schweinezucht und Schweinemast (SUS) (2), 36-39.
} 


\section{I.2 ZUR REPUTATION DER FLEISCHWIRTSCHAFT IN DER GESELLSCHAFT}

\section{I.2.1 Supply Chain Reputation in der Fleischwirtschaft}

\section{Autoren: Friederike Albersmeier und Achim Spiller}

Erschienen als „Diskussionsbeitrag 0811“ des Departments für Agrarökonomie und Rurale Entwicklung der Georg-August-Universität Göttingen, Göttingen, (2008).

In den vergangenen Jahren ist das Agribusiness vermehrt in den öffentlichen Fokus gerückt. Längst interessiert sich die Gesellschaft nicht mehr nur für Produktsicherheit und -qualität, sondern sukzessive auch für soziale, ethische und ökologische Aspekte. Durch die zahlreichen Skandale und Krisen gerät insbesondere die Fleischwirtschaft ins öffentliche Wahrnehmungsfeld. Aufgrund ihres hohen Einflusspotentials ist die Öffentlichkeit zu einem wichtigen Faktor für den Erfolg des einzelnen Unternehmens, aber auch insgesamt für die Fleischwirtschaft geworden. In dem gesellschaftlichen Diskurs hat es die Fleischbranche bislang nicht verstanden, sich in die Diskussionen einzubringen. In zahlreichen Studien konnte demgegenüber nachgewiesen werden, dass Firmen mit einer guten Reputation über dauerhafte Unterstützungspotentiale verfügen. Die Ergebnisse der präsentierten Studie zeigen ein sehr heterogenes Bild hinsichtlich der Reputation. Im Vergleich $\mathrm{zu}$ anderen Agrar- und Nicht-Agrarsektoren hat die Fleischwirtschaft den schlechtesten Ruf. Innerhalb der Branche treten starke Unterschiede zwischen den Wertschöpfungsstufen auf. Während die Landwirtschaft und der LEH ein recht hohes Maß an Vertrauen und Glaubwürdigkeit besitzen, wird die Reputation des Sektors vor allem durch den negativen Ruf der Schlachtunternehmen und Wursthersteller nachhaltig beeinflusst. Beide Stufen leiden unter einem massiven Kommunikationsdefizit. 


\section{I.2.2 Die Reputation der Fleischwirtschaft: Eine Kausalanalyse}

\section{Autoren: Friederike Albersmeier und Achim Spiller}

Eingereicht bei „Agrarwirtschaft - Zeitschrift für Betriebswirtschaft, Marktforschung und Agrarpolitik" im November 2009. Momentan befindet sich der Beitrag im ReviewProzess. ${ }^{8}$

Die Fleischwirtschaft steht zunehmend im Fokus der öffentlichen Diskussion. Die Unternehmen der Branche verhalten sich dabei eher passiv. Die Distanz zwischen Gesellschaft und der Fleischwirtschaft ist in den vergangenen Jahren daher größer geworden. Anzeichen für die mangelnde Akzeptanz sind z. B. Initiativen gegen Bauvorhaben, ein geringes Vertrauen und ein steigender Vegetarieranteil. Um Rückschlüsse auf das gesamte Ausmaß dieser Problematik zu gewinnen, wird in der vorliegenden Arbeit die Reputation der Fleischwirtschaft umfassend untersucht. Unter Verwendung der Partial Least Squares Methode (PLS) werden Einfluss- und Erklärungsgrößen analysiert. Die Ergebnisse zeigen, dass das Interesse und Wissen über die Branche insgesamt eher gering sind. Hierin begründet sich möglicherweise der hohe Einfluss der affektiven Dimensionen Zuverlässigkeit und Emotional Appeal, während die Erwartungen an die Erfüllung spezifischer Leistungskomponenten (wie z. B. Tierschutz) von untergeordneter Relevanz sind. Die Analysen verdeutlichen ferner die große Bedeutung der Reputation zur Erklärung verschiedener Akzeptanzprobleme der Branche.

\footnotetext{
${ }^{8}$ Dieser Beitrag wurde mit leicht verändertem Titel auf der 49. Jahrestagung der Gesellschaft für Wirtschafts- und Sozialwissenschaften des Landbaues e.V. (Gewisola) vom 30. September - 02.Oktober 2009 in Kiel vorgestellt. Als ein Beitrag von insgesamt dreien erhielt es den „Best Paper Award“ und die Möglichkeit, neben dem Tagungsband in der „Agrarwirtschaft“ veröffentlicht zu werden. Der Tagungsband befindet sich im Druck.
} 


\section{I.2.3 Das Ansehen der Fleischwirtschaft: Zur Bedeutung einer stufenübergreifenden Perspektive}

Autoren: Friederike Albersmeier und Achim Spiller

Erschienen in: Böhm, J., Albersmeier, F., Spiller, A. (Hrsg.): Die Ernährungswirtschaft im Scheinwerferlicht der Öffentlichkeit, Eul-Verlag, 213-250, (2009).

In den vergangenen Jahren haben die Probleme zwischen der Fleischwirtschaft und der Öffentlichkeit stetig zugenommen. Das Resultat dieser Konflikte ist eine mangelnde Akzeptanz in der Gesellschaft, die u. a. durch Bürgerinitiativen gegen Bauvorhaben und ein verringertes Vertrauen in die Fleischbranche zum Ausdruck kommt. Eine Befragung von knapp 700 Verbrauchern spiegelt das große Reputationsdefizit der Fleischwirtschaft wider. In einem zweiten Schritt wurden die Zusammenhänge und Auswirkungen der Reputation innerhalb der Wertschöpfungskette analysiert. Insbesondere der Einfluss der vor- und nachgelagerten Stufen sowie deren Effekte auf wichtige Akzeptanzfaktoren der Fleischwirtschaft konnten unter Verwendung der Partial Least Squares Methode (PLS) untersucht werden. Die Ergebnisse zeigen, dass die jeweils benachbarten Stufen erheblichen Einfluss auf die Reputation nehmen. Ein Reputationsmanagement kann daher nicht alleine von einer Prozessstufe der Fleischwirtschaft realisiert werden, sondern sollte stufenübergreifend angelegt sein: „one step up, one step down“. 


\section{I.2.4 Zur Wahrnehmung der Qualität von Schweinefleisch beim Kunden}

Autoren: Friederike Albersmeier, Daniel Mörlein und Achim Spiller

Erschienen als „Diskussionsbeitrag 0912“ des Departments für Agrarökonomie und Rurale Entwicklung der Georg-August-Universität Göttingen, Göttingen, (2009).

Die naturwissenschaftlich messbaren Aspekte der Qualität von Frischfleisch, wie der Hygienestatus, die Prozessqualität oder Nährwertgehalte, spielen in der konkreten Einkaufssituation für Kunden am Point of Sale kaum eine Rolle, weil sie nicht sichtbar bzw. nachvollziehbar sind. Vielmehr können sich die Kunden am Kühlregal nur auf wahrnehmbare Eigenschaften wie Farbe, Preis, Zuschnitt oder Marmorierung konzentrieren. Dasselbe Produkt erhält auf diese Weise völlig verschiedene Qualitätsbeurteilungen durch die subjektive Wahrnehmung eines jeden Betrachters.

Es ist daher von großem Interesse zu erfahren, wie die wahrgenommene Fleischqualität individuell bestimmt wird. Sie hängt dabei nur teilweise mit der tatsächlich messbaren Qualität zusammen und kann sich auch bei ein und derselben Person im Zeitablauf ändern. Verschiedene Studien konnten bereits zeigen, dass sich die Konsumenten durch ein hohes Maß an Unsicherheit bezüglich der Fleischqualität auszeichnen. Bisher ist jedoch wenig über die Qualitätswahrnehmung von Fleisch am Point of Sale bekannt. Um den Bedürfnissen und Anforderungen der Kunden gerecht zu werden, ist es für die Anbieter von Frischfleisch aber besonders wichtig zu wissen, welche Faktoren dabei entscheidend sind. Die folgende Studie widmet sich dieser Fragestellung. 


\title{
Teil II: Reputationsmanagement: Qualitätssicherung im Agribusiness
}

\section{II.0 Einleitung: Risikoorientierte Prüfung in Zertifizierungssyste- men der Land- und Ernährungswirtschaft}

\author{
Autoren: Holger Schulze, Friederike Albersmeier und Achim Spiller
}

Erschienen als „Diskussionsbeitrag 0607“ des Departments für Agrarökonomie und Rurale Entwicklung der Georg-August-Universität Göttingen, Göttingen, (2006).

Beiträge aus der Wirtschaftsprüfungspraxis zeigen, dass die Effizienz und Effektivität von Kontrollen entscheidend verbessert werden kann, wenn der Abschlussprüfer Kontrollen durchführt, die sich an der Risikolage und dem Risikopotential des einzelnen Unternehmens orientieren. Im Gegensatz $\mathrm{zu}$ dem heute in der Zertifizierung vorherrschenden „Gleichheitsgrundsatz“ geht es hierbei um die Konzentration der knappen Prüfungsressourcen auf kritische Fälle. Im Rahmen des Beitrags wird dieses Konzept auf die Qualitätssicherungssysteme der Land- und Ernährungswirtschaft übertragen und ein erster Überblick über die Grenzen und Potentiale sowie Ansätze zur Entwicklung eines risikoorientierten Steuerungstools aufgezeigt. 


\title{
II.1 BEWERTUNG VON QUALITÄTSSICHERUNGSSYSTEMEN
}

\section{II.1.1 The Reliability of Third-Party Certification in the Food Chain: From Checklists to Risk Oriented Auditing}

\author{
Autoren: Friederike Albersmeier, Holger Schulze, Gabriele Jahn und Achim Spiller
}

Erschienen in: Food Control 20(10), 927-935, (2009). ${ }^{9}$

In recent years, certification has become increasingly relevant for the agribusiness. In Europe, substantial parts of the value chain are already being certified by standards such as the International Food Standard (IFS) or EurepGap. It is not known, however, whether these approaches can de facto ensure a high quality control. This article is based on the database analysis of the German certification system Quality and Safety (QS) and a workshop with QS-certification bodies conducting $85 \%$ of all agricultural audits. It tries to deduce the first empirical hypotheses concerned with the connection between the reliability of third-party certification and the institutional framing of standards.

\footnotetext{
${ }^{9}$ Der Artikel wurde vorab in gekürzter und geänderter Version mit dem Titel „Checklist Governance? Zur Prüfungsqualität von Zertifizierungssystemen im Agribusiness“" auf der GEWISOLA- (Gesellschaft für Wirtschafts- und Sozialwissenschaften des Landbaus e.V.) Jahrestagung 2007 präsentiert und ist erschienen im Tagungsband „Good Governance in der Agrar- und Ernährungswirtschaft", Kuhlmann, F. und Schmitz, M. (Hrsg.), 215-225.

In geänderter Fassung und mit einem anderen Titel auch veröffentlicht als „Risikoorientierte Prüfung in Zertifizierungssystemen: Eine Auswertung der QS-Datenbank“ in: Zukunftsperspektiven der Fleischwirtschaft - Verbraucher, Märkte, Geschäftsbeziehungen, Spiller, A. und Schulze, B. (Hrsg.) Universitätsverlag, Göttingen (2008), 211-230.

In geänderten, kürzeren und längeren Fassungen sowie unter anderen Titeln wurde der Beitrag auf verschiedenen Konferenzen vorgestellt und in deren Tagungsbänden oder im Internet veröffentlicht:

- Schulze, H.; Albersmeier, F.; Jahn, G.; Spiller, A. (2006): Risk-oriented audits to improve the quality of certification standards in the food sector. In: Zazueta, F.; Xin, J.; Ninomiya, S.; Schiefer, G. (Hrsg.) (2006): Computers in Agriculture and Natural Resources, Proceedings of the 4th World Congress in Orlando, Florida USA, July 24-26, St. Joseph, 753-758.

- Schulze, H.; Albersmeier, F.; Spiller, A.; Jahn, G. (2006): Audit Risk Factors in Certification: How can risk-oriented audits improve the quality of certification standards? In: Proceedings zum 98th Seminar der European Association of Agricultural Economists (EAAE) "Marketing Dynamics within the Global Trading System: New Perspectives", 29. Juni-02. Juli 2006, Chania, Crete, Greece, 106.

- Schulze, H.; Albersmeier, F.; Spiller, A.; Jahn, G. (2006): Checklist Governance: Risk-oriented audits to improve the quality of certification standards in the food sector. In: Proceedings zum „16th Annual World Food and Agribusiness Forum, Symposium and Case Conference“ der International Food and Agribusiness Management Association (IAMA) "Agribusiness, Food, Health, and Nutrition", 10.-13. Juni 2006, Buenos Aires (Argentinien). URL: http://www.ifama.org/ conferences/2006_Conference/SymposiumFinal/ 1064_Paper.pdf.
} 


\section{II.1.2 Heterogeneity in the Evaluation of Quality Assurance Systems: The International Food Standard (IFS) in European Agribusiness}

\section{Autoren: Holger Schulze, Friederike Albersmeier, Jana-Christina Gawron, Achim Spiller und Ludwig Theuvsen}

Erschienen in: International Food and Agribusiness Management Review (IFAMR) 11(3), 99-138, (2008). ${ }^{10}$

Due to the growing demands of customers, particularly large retailers, and several food crises, such as BSE or Dioxin residues in feedstuff, quality assurance schemes have become more and more popular in the agricultural sector and the food industry. With the help of such systems, food safety, the transparency of the supply chain and, for this reason, the quality of food products should be improved. Furthermore, the advancement of the competitiveness of the European agribusiness parallels this development. With this trend in mind, it seems worthwhile to take a closer look at the implementation of certification schemes in Europe, especially at the evaluation of the participating companies. The study focuses on the International Food Standard (IFS), which has gained great relevance in the European food industry since all major retailers have been subscribing to the system and, for the most part, no longer accept suppliers that do not have IFS certificates. 389 food manufacturers answered an extensive questionnaire and gave insights into their perceptions of the advantages and disadvantages of the IFS. The results highlight the fact that the overall evaluation of the IFS is primarily affected by the perceived usefulness and the evaluation of the catalogue of requirements. Furthermore, a cluster analysis was conducted and three clusters were identified representing heterogeneous evaluations of the IFS.

\footnotetext{
${ }^{10}$ Dieser Beitrag wurde eingereicht für das 18th Annual World Forum and Symposium der IAMA (International Food and Agribusiness Management Association) vom 14.-17. Juni 2008 in Monterey, California, USA und als einer der 20 besten Beiträge von 135 für den „Best Paper Award“ nominiert. Die 10 besten Paper erschienen im "International Food and Agribusiness Management Review” (IFAMR).
} 


\section{II.1.3 Evaluation and reliability of the organic certification system: perceptions by farmers in Latin America}

Autoren: Friederike Albersmeier, Holger Schulze und Achim Spiller

Erschienen in: Sustainable Development 17(5), 311-324, (2009). ${ }^{11}$

In recent years, organic agriculture has developed rapidly, and organic certification systems are now used worldwide. However, if these standards are unable to meet their promises, their reliability will be called into question and trust in organic products will fade. As major suppliers of organic food to the European market, it is crucial for Latin American farmers to show their customers that the standards they have implemented are actually delivering what they promise. Considering the manifold resources that are being poured into these systems, it seems reasonable to critically review their reliability. A central indicator for the reliability of the system is the overall evaluation of organic certification. Therefore, it is the objective to analyze the relationship of both constructs. Findings show that most farmers are satisfied with the organic certification system and believe in its reliability; however, they fear the costs of bureaucracy and documentation.

${ }^{11}$ Dieser Beitrag wurde vorgestellt auf der 14. Annual International Sustainable Development Research Konferenz vom 21.-23. September 2008 in Neu Delhi (Indien) und in leicht veränderter Form veröffentlicht in den Proceedings zur Konferenz. 


\section{II.2 ENTWICKLUNG EINES MANAGEMENT-ToOLS ZUR ABSICHERUNG VOR REPUTATIONSSCHÄDEN}

\section{II.2.1 System Dynamics in Quality Certifications: Development of an Audit Integrity System}

Autoren: Friederike Albersmeier, Holger Schulze und Achim Spiller

Erschienen in: International Journal on Food System Dynamics 1(1), 69-81, (2010). ${ }^{12}$

Due to the complex structure of certification schemes the risk of flaws and scandals is generally high. It has further increased by several developments during the last years. With regard to their potential effects, it is questionable whether the certification approaches are actually able to detect deficiencies within the system and thus prevent crises which may lead to its breakdown. Hence, the ability of a standard to meet its objectives of food quality and safety needs to be enforced. In this contribution we launch the implementation of a controlling tool which automatically monitors audit quality based on information of the respective data bases. By analysing possible negative influences, opportunistic behaviour can thus be detected.

\footnotetext{
12 Dieser Beitrag wurde in leicht geänderter Version vorgestellt als contributed paper auf dem „1st International European Forum on Innovation and System Dynamics in Food Networks", vom 15.-17. Februar 2007 in Innsbruck-Igls, Österreich und ist erschienen im Tagungsband „Innovation and System Dynamics in Food Networks 2007“, Rickert, U. und Schiefer, G. (Hrsg.) 11-22, (2007).
} 


\title{
II.2.2 The Application of Databases as an Information Management Tool in Quality Certification Systems
}

\author{
Autoren: Holger Schulze, Friederike Albersmeier und Achim Spiller
}

Dieser Beitrag wurde vorgestellt als contributed paper auf der „EFITA/WCCA 2007 6th Biennial Conference of the European Federation of IT in Agriculture - Environmental \& Rural Sustainability through ICT”, vom 02.-05. Juli 2007 in Glasgow, Schottland und ist erschienen auf der CD zur Konferenz, Parker, C.; Skerratt, S.; Park, C. und Shields, J. (Hrsg.), (2007).

While quality assurance systems in the agribusiness are constantly growing, there have been few investigations as to whether these approaches can actually ensure a high quality control. Since certification procedures are applied as a tool to increase trust and confidence within the agri-food chain, a high audit quality is an essential element for certification standards. Thus, the necessity of regulating the audit quality is ranked high by the scheme owners and up to now appropriate tools have hardly been developed. Hence, this contribution is focused on the development of an information management tool in quality certification systems. The results indicate that the application of such an audit quality controlling tool in practice offers a possibility to achieve detailed information about the quality assurance system. Thus, the validity and reliability of audit control can be optimised continuously. By means of this instrument, a risk oriented audit is supported enabling us to calculate the optimal control interval and depth for each business, an application of unannounced sampling audits and a differentiated priority of inspection contents. 


\title{
Teil III: Exkurs: Beziehungsmanagement auf dem Landpachtmarkt
}

\section{III.1 Die Bedeutung der Pachtpreisanpassungsklausel als Instrument des Risikomanagements}

\author{
Autoren: Cord-Herwig Plumeyer, Friederike Albersmeier, Ludwig Theuvsen und \\ Birgit Schulze
}

Dieser Beitrag wurde vorgestellt auf der 19. Jahrestagung der Österreichischen Gesellschaft für Agrarökonomie (ÖGA) vom 24.-25. September 2009 in Innsbruck, Österreich und wurde im September 2009 zur Veröffentlichung im ÖGA-Jahrbuch eingereicht.

Am Landpachtmarkt nimmt der Wettbewerb um landwirtschaftliche Nutzflächen stetig zu. Um in Zeiten volatiler Märkte ein kalkulierbares und stabiles Verhältnis zwischen Pächtern und Verpächtern zu gewährleisten, werden Pachtpreisanpassungsklauseln (PPAK) von der Wissenschaft als Risikomanagementinstrument für beide Vertragsparteien vorgeschlagen. Auf Basis einer empirischen Studie werden der Status quo sowie die Akzeptanz der PPAK bei Pächtern analysiert. Die Ergebnisse offenbaren eine noch vergleichsweise geringe Verbreitung der PPAK sowie signifikante Einstellungsunterschiede zwischen Nutzern und Nicht-Nutzern. Dies liefert wichtige Ansatzpunkte zur Förderung der weiteren Verbreitung von PPAK. 


\title{
III.2 Die Bedeutung von Pachtpreisanpassungsklauseln auf dem Landpachtmarkt: Eine empirische Analyse von Verpächtern und Pächtern
}

\author{
Autoren: Cord-Herwig Plumeyer, Friederike Albersmeier, Ludwig Theuvsen und \\ Birgit Schulze
}

Eingereicht bei „Agrarwirtschaft - Zeitschrift für Betriebswirtschaft, Marktforschung und Agrarpolitik“ im November 2009.

Auf dem Landpachtmarkt nimmt der Wettbewerb um landwirtschaftliche Nutzflächen stetig zu. Neuere Entwicklungen wie die politische Förderung der Bioenergieproduktion haben diesen Trend weiter forciert. Um in Zeiten volatiler Märkte ein stabiles Verhältnis zwischen Pächtern und Verpächtern zu gewährleisten, sind Pachtpreisanpassungsklauseln (PPAK) ein in der Fachpresse viel diskutiertes Risikomanagementinstrument. Um den Status quo sowie die Akzeptanz von PPAK in Pachtverträgen zu analysieren, wurden im März 2009 zwei empirische Studien bei Pächtern sowie Verpächtern in Niedersachsen durchgeführt. Die Ergebnisse zeigen, dass PPAK bislang nur in wenigen Pachtverträgen verankert sind. Zwischen Nutzern und Nicht-Nutzern können deutliche Unterschiede in der Einstellung zu PPAK aufgedeckt werden. Neben der Einstellung beeinflussen weitere Faktoren die Implementierung derartiger Klauseln - die Bedeutung dieser Faktoren wird mittels einer multinomialen logistischen Regression analysiert. Hierdurch lassen sich wichtige Ansatzpunkte für die Beseitigung von Schwachstellen bei den bestehenden PPAK aufzeigen und Strategien für eine bessere Akzeptanz bei Pächtern und Verpächtern ableiten. 


\section{Literatur}

Abraham, M., Arpagaus, J., Michels, V. (2005): Die Entstehung von Reputation in sozialen Systemen: Eine Projektskizze. Arbeitspapier des Institutes für Soziologie der Universität Bern.

Albersmeier, F., Spiller, A. (2009): Die Reputation der Fleischwirtschaft in der Gesellschaft: Eine Kausalanalyse. (angenommen zur Veröffentlichung im GEWISOLATagungsband 2009).

Albersmeier, F., Spiller, A., Jäckel, K. (2008): Öffentlichkeitsorientierung in der Ernährungswirtschaft: Eine empirische Studie zum Umgang mit kritischen Anspruchsgruppen. In: Zeitschrift für Management 3 (4), 363-383.

Antle, J.M. (2001): Economic analysis of food safety. In B. Gardner, \& G. Rausser, Handbook of agricultural economics - Marketing, Distribution and Consumers. Amsterdam, 1084-1136.

Aron, D.J. (1987): Worker Reputation and Productivity Incentives. In: Journal of Labor Economics 5 (4), Part 2, 87-106.

Auriol, E., Schilizzi, S.G.M. (2002): Quality signaling through certification, Theory and an application to agricultural seed markets, University of Toulouse, Toulouse.

Böcker, A., Bredahl, M.E., Northen, J. (2003): ISO 9000 certification in British agribusiness: motivations and performance impacts. In: Schiefer, G.; Rickert, U. (Hrsg.) (2003): Quality Assurance, Risk Management and Environmental Control in Agriculture and Food Supply Networks. Proceedings of the 82nd Seminar of the European Association of Agricultural Economists (EAAE), University of Bonn, Bonn, Germany, 51-60.

Bodenstein, G., Spiller, A. (1998): Marketing: Strategien, Instrumente, Organisation, Landsberg/Lech.

Caswell, J.A., Mojduszka, E.M. (1996): Using informational labeling to influence the market for quality in food products. Working paper. American Journal of Agricultural Economics, 78(5), 1248-1253.

Chung, S.Y., Schneeweis, T., Eneroth, K. (1999): The UK and US expertise, SSRN Electronic, Paper Collection.

Coombs, W.T., Holladay, S.J. (2006): Unpacking the halo effect: reputation and crisis management. In: Journal of Communication Management 10 (2), 123-137.

Cordeiro, J.J., Sambharya, R.B. (1997): Do corporate reputations influence security analyst earnings forecast? An empirical study. In: Corporate Reputation Review 1 (1/2), 94-97.

Davies, G., Chun, R., Vinhas da Silva, R., Roper, S. (2003): Corporate Reputation and Competiveness, London.

Deephouse, D.L. (1997): The effect of financial and media reputations on performance. In: Corporate Reputation Review 1 (1/2), 68-71.

Du, S., Bhattacharya, C.B., Sanker, S. (2007): Reaping relational reward from corporate social responsibility: The role of competitive positioning. In: International Journal of Research in Marketing 24 (3), 224-241. 
Dunbar, R.L.M., Schwalbach, J. (2000): Corporate reputation and performance in Germany. In: Corporate Reputation Review 3 (2), 115-123.

Eccles, R.G., Newquist, S.C., Schatz, R. (2007): Reputation and its risks. In: Harvard Buisness Review, Februar, 104-114.

Fombrun, C.J., Shanley, M. (1990): What's in a name? Reputation building and corporate strategy. In: Academy of Management Journal 33 (1), 233-258.

Fombrun, C.J., van Riel, C.B.M. (2003): Fame \& Fortune: How Successful Companies Build Winning Reputations, FT Press.

Fuente Sabate, J.M. de la, Quevedo Puente, E. de (2003): Empirical Analysis of the Relationship between Corporate Reputation and Financial Performance: A Survey of the Literature. In: Corporate Reputation Review 6 (2), 161-177.

Fulponi, L. (2006): Private voluntary standards in the food system: the perspective of major food retailers in OECD countries. In: Food Policy, 31 (1), 1-13.

Golan, E., Kuchler, F., Mitchell, L., Greene, C., Jessup, A. (2001): Economics of Food Labeling. In: Journal of Consumer Policy 24 (2), 117-184.

Gray, E.R., Balmer, J.M.T. (1998): Managing Corporate Image and Corporate Reputation. In: Long Range Planning 31 (5), 695-702.

Hammond, S.A., Slocum, J.W. (1996): The impact of prior firm financial performance on subsequent corporate reputation. In: Journal of Business Ethics 15, 159-165.

Hatanaka, M., Bain, C., Busch, L. (2005): Third-Party Certification in the Global Agrifood System. In: Food Policy 30 (3), 354-369.

Jahn, G., Schramm, M., Spiller, A. (2005): The Reliability of Certification: Quality Labels as a Consumer Policy Tool. In: Journal of Consumer Policy 28 (1), 53-73.

Jahn, G., Spiller, A. (2005): Controversial positions about the QS System in agriculture: an empirical study. In: Theuvsen, L., Spiller, A., Peupert, M., Jahn, G. (Hrsg.): Quality Management in Food Chains, Wageningen Academic Publishers, 355368.

Jones, G., Jones, B., Little, P. (2000): Reputation as a reservoir: Buffering against loss in times of economic crisis. In: Corporate Reputation Review 3 (1), 21-29.

Luning, P.A., Marcelis, W.J. (2005): Food quality management and innovation. In: Jongen, W.M.F.; Meulenberg, M.T.G. (Hrsg.) Innovation in agri-food systems: product quality and consumer acceptance, Wageningen, 293-341.

Mahon, J.F. (2002): Corporate Reputation: Research Agenda Using Strategy and Stakeholder Literature. In: Business \& Society 41 (4), 415-445.

Mast, C., Huck, S., Güller, K. (2005): Kundenkommunikation, Stuttgart.

McGuire, J.B., Schneeweis, T., Branch, B. (1990): Perceptions of firm quality: A cause or result of firm performance? In: Journal of Management 16 (1), 167-180.

Pennings, J.M.E, Wansinka, B., Meulenberg, M.T.G. (2002): A note on modeling consumer reactions to a crisis: The case of the mad cow disease. In: International Journal of Research in Marketing 19 (1), 91-100.

Poppe, C., Kjærnes, U. (2003): Trust in food in Europe: A Comparative Analysis, National institute for consumer research, Oslo. 
Riahi-Belkaoui, A., Pavlik, E. (1991): Asset management performance and reputation building for large US firms. In: British Journal of Management 2, 231-238.

Roberts, P.W., Dowling, G.R. (1997): The value of a firm's corporate reputation: How reputation helps attain and sustain superior profitability. In: Corporate Reputation Review 1 (1/2), 72-75.

Schlecht, S., Albersmeier, F., Spiller, A. (2008): Konflikte bei landwirtschaftlichen Stallbauprojekten: Eine empirische Untersuchung zum Bedrohungspotential kritischer Stakeholder. In: Diskussionsbeitrag 0808 des Departments für Agrarökonomie und Rurale Entwicklung der Georg-August-Universität Göttingen.

Schulze, H., Albersmeier F., Spiller, A. (2006): Risikoorientierte Prüfung in Zertifizierungssystemen der Land- und Ernährungswirtschaft. In: Diskussionsbeitrag 0607 des Departments für Agrarökonomie und Rurale Entwicklung der GeorgAugust-Universität Göttingen, Göttingen.

Smith, N.C. (2003): Corporate Social Responsibility - Whether or how? In: California Management Review 45 (4), 52-76.

Sobol, M.G., Farrelly, G. (1988): Corporate reputation: A function of relative size or financial performance? In: Review of Business and Economic Research 24, 45-59.

Srivastava, R.K., McInish, T.H., Wood, R.A., Capraro, A.J. (1997): The value of corporate reputation: Evidence from equity markets. In: Corporate Reputation Review 1 (1), 62-67.

Theuvsen, L., Spiller, A. (2007): Perspectives of quality management in modern agribusiness. In: Theuvsen, L., Spiller, A., Peupert, M., Jahn, G. (Hrsg.): Quality Management in Food Chains, Wageningen Academic Publishers, 13-19.

Tuncer, B. (2001): From Farm to Fork? Means of Assuring Food Quality. Master Thesis, University of Lund, Sweden. URL: http://www.iiiee.lu.se/C1256B88002 B16EB/\$webA11/1E9E24A162052480C1256BE9002D 8FFA?OpenDocument.

Vergin, R.C., Qoronfleh, M.W. (1998): Corporate reputation and the stock market. In: Business Horizons 41 (1), 19-26.

Wiedmann, K.-P., Fombrun, C.J., van Riel, C.B.M. (2006): Ansatzpunkte zur Messung der Reputation von Unternehmen. In: Der Markt 45 (177), 98-109. 


\section{Resümee}

\section{Fazit}

Der Schwerpunkt der vorliegenden Dissertation liegt auf der Analyse der Beziehung zwischen der Öffentlichkeit und dem Agribusiness. Die Studien zeigen das Gefahrenpotential auf, welches von der Gesellschaft für die Branche ausgeht, und pointieren die Notwendigkeit des institutionellen Aufbaus und der inhaltlichen Konzeption eines strategischen Public Affairs-Managements. Vor dem Hintergrund der zukünftigen Herausforderungen gewinnen die Bereiche Öffentlichkeitsarbeit und Kommunikation erheblich an unternehmerischer Relevanz.

Land- und Ernährungswirtschaft werden sich künftig mit zahlreichen gesellschaftsrelevanten Themen auseinandersetzen müssen. Insbesondere die Klimadiskussion ist eine der vordinglichsten Problemstellungen für die Branche. Im Rahmen dieser Debatte wird der Ausstoß von klimaschädlichen Treibhausgasen - vorrangig aus der Tierproduktion angeprangert (BMVEL, 2005; FAO, 2006). Dieses Beispiel zeigt auch sehr anschaulich den Zielkonflikt zwischen der Gewinnung von (Bio-)Energie und der Tierernährung auf der einen Seite sowie der Versorgung der Gesellschaft mit Lebensmitteln auf der anderen Seite auf. Eng mit diesem Thema verbunden ist die Auseinandersetzung darüber, wie eine Weltbevölkerung von bald 7 Mrd. Menschen ernährt werden soll. Im Kontrast hierzu steht die vor allem in den Industriestaaten geführte Gesundheitsdiskussion, die den zu hohen Konsum von Fleisch kritisiert. Als Folge lässt sich bereits heute ein abnehmender Fleischverzehr und ein steigender Anteil an Vegetariern und Low-Meat-Consumern in Deutschland erkennen (Karmasin, 2001; GURATH, 2008; Schulze und Spiller, 2008).

Die Kritik an intensiven Haltungsformen sowie die Diskussion um Tierschutzstandards wird auch zukünftig nicht an Relevanz verlieren, denn grundsätzlich weisen etwa $20 \%$ der deutschen Bevölkerung ein gesteigertes Interesse für den Tierschutz auf (Schulze et al., 2008a). Bereits die in den letzten Monaten hitzig geführte Debatte um die betäubungslose Ferkelkastration hat die große Bedeutung dieses Themas für die Gesellschaft aufgezeigt. Breit diskutiert werden darüber hinaus auch die Arbeitsbedingungen für Mitarbeiter im Agribusiness. Diskussionspunkte sind hierbei z. B. Mindestlöhne, bessere Arbeitsbedingungen sowie die Abschaffung von Überwachungssystemen. 
Die vorliegende Dissertation setzt an den aufgezeigten gesellschaftlichen Konfliktpotentialen des Agribusiness an. Ziel ist es dabei, sowohl die Ursachen einer geringen Öffentlichkeitsorientierung als auch die Konsequenzen einer schlechten Reputation der Branche in der Gesellschaft auf materieller und sozialer Ebene herauszuarbeiten. Das in Abbildung 1 dargestellte Analysemodell skizziert die maßgeblichen Einflussfaktoren auf das Ansehen des Agrarsektors. Anhand der Darstellung werden im Nachfolgenden die Ursachen für das negative Ansehen der Agrarbranche stellvertretend am Beispiel der Fleischwirtschaft untersucht.

Abbildung 1: Analysemodell

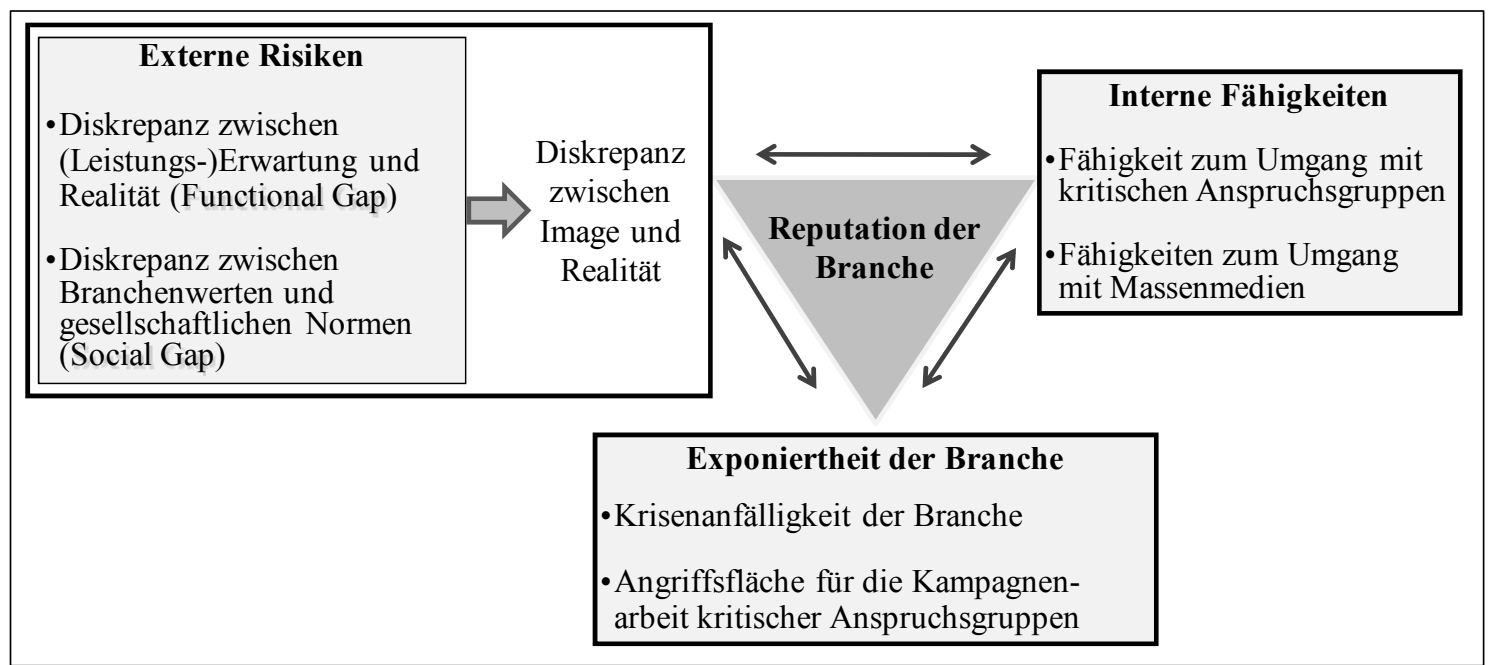

Quelle: Eigene Darstellung

Die Analyse der Gründe für die zunehmende Zahl an Konflikten zwischen der Gesellschaft und der Agrarbranche gestaltet sich vielschichtig und komplex. Die gewachsene öffentliche Exponiertheit - die Tatsache, dass die Unternehmen der Branche durch ihre Aktivitäten öffentliches Interesse tangieren bzw. selbst von der Öffentlichkeit beeinflusst werden (Dyllick, 1989) - ist dabei eine maßgebliche Ursache für die vielfältigen öffentlichen Auseinandersetzungen und Akzeptanzkonflikte sowie die Skandalisierung von Problemen in der Land- und Ernährungswirtschaft durch die Medien. Die Kritik und die Erwartungen, die an die Unternehmen des Agribusiness insgesamt gerichtet werden, sind oftmals sehr speziell auf eine Subbranche gerichtet und daher nicht über den gesamten Agrarsektor generalisierungsfähig (vgl. I.1.1). Einzelne Branchen - wie die Fleischbranche - sind von den öffentlichen Diskursen stärker betroffen als andere und verfügen infolge über ein vergleichsweise schlechteres Ansehen in der Gesellschaft. Insgesamt kann daher konstatiert werden, dass das Agribusiness von der Öffentlichkeit sehr heterogen wahrgenommen wird (vgl. I.2.1). Wie eine neue Studie der Wirt- 
schaftsWoche, die in Zusammenarbeit mit dem Wirtschaftsverband der führenden PRAgenturen und TNS Emnid erstellt wurde, jedoch zeigt, wird der Lebensmittelbranche als Ganzes im Vergleich mit sieben anderen deutschen Wirtschaftsbereichen die höchste Glaubwürdigkeit eingeräumt (Mai, 2009).

Die Auswirkungen einer schlechten Reputation sind derweil beachtlich. Die vorliegende Dissertation konnte aufzeigen, dass der defizitäre Ruf der Fleischwirtschaft neben der Standortakzeptanz, dem politischen Kontrolldruck, dem gesellschaftlichen Protest und der wahrgenommenen Attraktivität als Arbeitgeber auch den Fleischkonsum und das Vertrauen in Fleisch nachhaltig negativ beeinflusst (vgl. Kap. I.2.1).

Während die starke öffentliche Exponiertheit des Fleischsektors eine Grundlage für die schlechte Reputation der Branche bildet, so sind es nicht zuletzt auch die schwach ausgeprägten internen Fähigkeiten im Umgang mit Kritikern und den Massenmedien, die zu einem negativen Ruf beigetragen haben. Die vielfältigen gesellschaftlichen Diskussionslinien treffen auf eine Branche, die sich heute wenig professionell mit öffentlicher Kritik auseinandergesetzt hat. Die wenigsten Unternehmen verfügen über die institutionellen Einheiten und somit über die Möglichkeit, relevante Issues systematisch und vor allem frühzeitig zu identifizieren und auf diese Weise potentiellen Konfliktthemen proaktiv entgegenzuwirken. Die Branche hat es größtenteils nicht verstanden, eine effektive Kommunikationsstrategie und Krisen-PR aufzubauen, die vor Reputationsschäden schützen (vgl. I.0; Schulze et al., 2008b). Oftmals fehlt es bereits an den Grundvoraussetzungen hierfür, nämlich einer entsprechenden Sensibilität und einer aktiven Partizipation in den öffentlichen Debatten.

Diese Passivität wiederum fördert eine Medienberichterstattung, die meist ein negatives Bild des Fleischsektors zeichnet. Die Medien erlangen durch die Abtauch- oder LowProfile-Strategie der Branche ein erhebliches Einflusspotential auf die öffentliche Meinung und können auf diese Weise die öffentliche Wahrnehmung und das Vertrauen in Fleisch und den dahinterstehenden Sektor entscheidend steuern (Bruhn, 2008). Weitgehend wurde das Diskussionsfeld somit Kritikern, Nichtregierungsorganisationen (NGOs) und nicht zuletzt den Medien überlassen (vgl. I.0). Auf diese Weise konnte das Agribusiness in der Vergangenheit nicht den primär sozialmoralisch argumentierenden Kritiker-Gruppen entgegenwirken, um Krisen zu verhindern (Herger, 2006; Weiner, 2006). 
Die Disparität zwischen der Branchenkultur und den Normen und Werten der Gesellschaft lässt sich auch als Social Gap beschreiben. Die soziale Erwartungslücke ist in den letzten Jahren stetig gewachsen und spiegelt sich in der Kultur der Ernährungs- und insbesondere Fleischbranche wider. Diese ist maßgeblich durch eine negative Wahrnehmung der Gesellschaft und geringe Öffentlichkeitsorientierung geprägt (vgl. I.1.1/2). Die Schließung der kulturellen Lücke ist jedoch ein sehr komplexer Prozess und setzt ein strategisches und langfristig orientiertes Change-Management in den Unternehmen der Branche voraus (Spiller, 2009). Diese Herausforderung ist dabei weit größer und schwieriger zu überwinden als die Schließung der funktionalen Lücke, d. h. der Diskrepanz zwischen der materiellen Leistungserwartung der Öffentlichkeit und der Leistungserfüllung durch die Fleischwirtschaft (vgl. Abbildung 1).

Das Problem der funktionalen Lücke ist dabei grundsätzlich geprägt durch die Schwierigkeit der Evaluation der eigentlichen Ist-Komponente durch die Öffentlichkeit. Hierfür gibt es zwei wesentliche Ursachen: Zum einen fehlen der Gesellschaft infolge der zunehmenden Entfremdung von der Urproduktion in weiten Teilen die grundlegenden Fähigkeiten der Leistungsbewertung und zum anderen wird die Beurteilung durch die hohe Informationsasymmetrie und Intransparenz in der Supply Chain erschwert. Als ein Instrument zur Überbrückung dieses Informations-Ungleichgewichtes zwischen Gesellschaft und Agribusiness wurden von der Branche - wenn auch nicht ganz freiwillig - Zertifizierungsstandards implementiert. Diese überwachen entlang der (Fleisch-)Wertschöpfungskette verschiedene Prozesseigenschaften wie bspw. die Qualität und Sicherheit der Erzeugnisse. Entsprechende Labels und Zertifikate sollen dem Verbraucher als glaubwürdiges Qualitätssignal dienen, auf deren Basis (Kauf-) Entscheidungen und Produktbewertungen getroffen werden können.

Zusammenfassend lässt sich festhalten, dass die in der vorliegenden Dissertation präsentierten Studien die Bedeutung der Reputation und eines professionellen BeziehungsManagements für (kritische) Anspruchsgruppen und Medien unterstreichen. Denn nur Unternehmen bzw. eine Branche mit einem hohen Ansehen kann in der Öffentlichkeit insgesamt, oder im Speziellen bei NGOs oder Bürgerinitiativen, verstärkt Einfluss auf den Verlauf gesellschaftlicher Themen nehmen, Loyalität und Akzeptanz schaffen und infolgedessen Krisensituationen besser meistern (Gray und Balmer, 1998; Eisenegger und Künstle, 2003; Mast et al., 2005). Das Management der Reputation muss dabei durch eine effektive Kommunikationsstrategie und Krisen-PR begleitet werden. Auch 
die Maßnahmen zur Reduzierung der funktionalen Erwartungslücke (Beispiel: Qualitätssicherung) können langfristig nur dann erfolgreich sein, wenn diese kommunikativ unterstützt werden. Kritische Kampagnen von NGOs dürfen nicht ausgesessen, sondern müssen von der Branche bzw. den Verantwortlichen kommunikativ abgesichert werden. Die bislang verfolgte Low-Profile Strategie der Fleischwirtschaft stellt ein erhebliches latentes Risiko für die funktional durchaus erfolgreichen (Zertifizierungs-) Systeme dar.

Mit Blick auf die Praxis kann resümiert werden, dass die sehr stark operativ, auf das Tagesgeschäft ausgerichtete Fleischbranche vor der großen Herausforderung steht, strategische und langfristig angelegte PR-Konzepte, die die gesellschaftliche Akzeptanz dauerhaft sichern, zu erarbeiten und insbesondere auch erfolgreich in den Unternehmen $\mathrm{zu}$ implementieren. Auf der funktionalen Seite ist der Branche dies durch den Aufbau von Qualitätssicherungssystemen gelungen, im Speziellen fehlt es jedoch auch hier an Maßnahmen, die proaktiv vor Krisen schützen (wie bspw. das von den Autoren aufgezeigte Audit Integrity System).

Bislang wird gesellschaftlichen Ansprüchen von der Branche nur eine geringe Legitimation zugesprochen. Öffentlichkeit und insbesondere NGOs und Bürgerinitiativen werden vielmehr als ein lästiges Ärgernis und Störfaktoren gesehen, denkbare Chancen werden indessen nicht erkannt. Intangible, weiche Faktoren, wie die Reputation, oder gar altruistische Motive scheinen in der „Tough-guy macho culture“ der Fleischwirtschaft, die durch „schnelle Feedbacks und hohes Risiko bei ausgeprägter Kurzfristorientierung“ (Spiller, 2009: 357) geprägt ist, nur eine bedingte Rolle zu spielen.

Auch wenn die Wahrnehmung des gesellschaftlichen Drucks durch die Branche infolge der zunehmend härter werdenden Angriffe von NGO (Strafanzeigen, Einbrüche in Produktionsanlagen, heimliche Videoaufnahmen) (Engel, 2009) in der letzten Zeit gestiegen ist, so erfolgte bislang noch kein strategisches Umdenken. Die Sensibilisierung konnte darüber hinaus auch im schnelllebigen Tagesgeschäft keine grundlegenden Veränderungen hervorrufen. In der Fleischwirtschaft sind weiter die auf Kostenführerschaft ausgerichteten Unternehmensstrategien vorherrschend und eine stärkere Öffentlichkeitsorientierung wurde bisher nicht nachdrücklich angestrebt.

Während die im Rahmen der vorliegenden Dissertation durchgeführten wissenschaftlichen Untersuchungen diese Problemstellung erstmals für den Agrarsektor - und im Speziellen für die Fleischwirtschaft - aufgreifen und den Status quo untersuchen, 
müssen in einen nachfolgendem Schritt darauf aufbauend entsprechende Konzepte erarbeitet werden. Insgesamt verweisen die Ergebnisse der Arbeiten hierbei auf den hohen Stellenwert, den ein professionelles Kommunikations-Management in diesem Zusammenhang einnimmt. Nicht nur die emotionale, auf Vertrauen und Glaubwürdigkeit basierende Seite der Reputation muss dabei durch kommunikative Maßnahmen gestärkt werden, sondern vielmehr auch die funktionalen Aspekte. Insbesondere das Beispiel der Qualitätssicherung hat gezeigt, dass Glaubwürdigkeit und Vertrauen entscheidend sind. Eine maßgebliche Beeinträchtigung für den Aufbau von Vertrauen, Zuverlässigkeit und Glaubwürdigkeit stellt hierbei jedoch insbesondere die Diskrepanz zwischen den Werten und Normen der Gesellschaft und denen der Fleischbranche (Social Gap) dar.

\section{Ausblick: Herausforderungen für Wissenschaft und Praxis}

Die Herausforderung für die Branche wird darin liegen, zum einen die Sensibilität gegenüber Anspruchsgruppen und ihren Forderungen zu erhöhen, auf diese zu reagieren und nach Abwägung aller Faktoren in ihren Entscheidungsprozessen zu berücksichtigen (vgl. I.1.1; Bruhn, 2008). Zum anderen sind diese Entscheidungsprozesse in ein integriertes Beziehungs-Management einzubetten. Maßgeblich hierfür ist jedoch, dass eine sichtbare Veränderung nicht nur für die Unternehmens- bzw. Branchenumfeld - insbesondere für die Öffentlichkeit - sichtbar wird, sondern diese Neugestaltung auch intern getragen wird und sich dort konsequent fortsetzt. Dies setzt zunächst einen internen Wandel, d. h. eine Erweiterung der Branchenkultur um die Dimension der Öffentlichkeitsorientierung voraus. Ohne diese Umstrukturierung werden Maßnahmen, die nach außen zielen, nur Potemkin-Dörfer sein, die von Kritikern leicht enttarnt werden können und entsprechend nicht nachhaltig gestaltet sind. Der Veränderungsprozess muss vielmehr phasenweise und über mehrere Jahre angelegt sein. Abbildung 2 gibt ein vereinfachtes Modell der Veränderungsprozesse wieder. 
Abbildung 2: Managementmaßnahmen und ihre internen und externen Ziele

\begin{tabular}{|c|c|c|}
\hline $\begin{array}{c}\text { Interne Maßnahmen } \\
\text { und Ziele }\end{array}$ & $\begin{array}{l}1 \\
\vdots \\
\vdots \\
\vdots\end{array}$ & Externe Ziele \\
\hline $\begin{array}{l}\text { Maßnahme: Wandel der } \\
\text { Branchenkultur } \\
\text { Ziel: Stärkere Öffentlichkeits- } \\
\text { orientierung }\end{array}$ & $\begin{array}{c}\vdots \\
\vdots \\
\vdots \\
\vdots \\
\vdots \\
\vdots \\
\vdots\end{array}$ & \multirow[b]{2}{*}{$\begin{array}{l}\text { Ziel: Schaffung von Glaubwür- } \\
\text { digkeit und Vertrauen }\end{array}$} \\
\hline $\begin{array}{l}\text { Maßnahmen: } \\
\text { 1. Erarbeitung von PR-und }\end{array}$ & $\stackrel{\vdots}{\stackrel{1}{\prime}}$ & \\
\hline Kommunikationsstrategien & \multirow{4}{*}{$\begin{array}{c}\vdots \\
\vdots \\
\vdots \\
\vdots \\
\vdots \\
\vdots \\
\vdots \\
\end{array}$} & \multirow[b]{5}{*}{$\begin{array}{l}\text { Ziel: Verbesserung und } \\
\text { Absicherung der Reputation }\end{array}$} \\
\hline 2. Aufbau eines professionellen & & \\
\hline Beziehungs-Managements zur & & \\
\hline Öffentlichkeit & & \\
\hline $\begin{array}{l}\text { Maßnahme: Aufbau eines } \\
\text { professionellen Reputations- }\end{array}$ & $\underset{i}{\stackrel{!}{a}}$ & \\
\hline Managements & $\vdots$ & \\
\hline & $\begin{array}{c}\vdots \\
\vdots \\
\vdots \\
\vdots \\
\vdots\end{array}$ & $\begin{array}{c}\text { Erhöhungder } \\
\text { gesellschaftlichen Akzeptanz }\end{array}$ \\
\hline
\end{tabular}

Quelle: Eigene Darstellung

Vorrangiges Ziel für die gesamte Branche ist zunächst der Aufbau von Glaubwürdigkeit und Vertrauen, um darauf basierend eine PR- und Kommunikationsstrategie und insbesondere ein Reputationsmanagement zu entwickeln. In dessen Folge kann gesellschaftliche Akzeptanz für das wirtschaftliche Handeln der Branche generiert werden. Eine maßgebliche Voraussetzung hierfür ist eine intakte Reputation. Dabei müssen Reputationsmanagement und PR-Konzepte ineinander greifen.

Basierend auf den Erfahrungen, die die Autorin insbesondere mit der Schlachtbranche sammeln konnte, scheinen die Unternehmen der Branche bislang - aus den bereits zuvor genannten Gründen - noch nicht bereit, sich mit derartigen Fragestellungen intensiv auseinanderzusetzen. Während die vorliegende Arbeit erste Ansätze für den Wandlungsprozess liefert, bleibt die Frage nach umsetzbaren und gezielten Strategien zur Verbesserung der Reputation weiter offen. Zum einen müssen von der wissenschaftlichen Seite weitergehende und auf die Fleischwirtschaft zugeschnittene Konzepte erarbeitet werden, die insbesondere bei der Veränderung der Branchenkultur als auch bei dem Aufbau bzw. der Optimierung der strategischen Einheiten zur Öffentlichkeitsarbeit und Kommunikation und dem Reputationsmanagement ansetzen. Zum anderen muss vor allem auch die Branche den gesellschaftlichen Willen für sich als Chance begreifen. Dabei besteht das grundsätzliche Dilemma, dass der Fleischsektor bislang 
Entscheidungen vorrangig auf Basis monetärer Überlegungen bzw. auf Basis externen Drucks (z. B. durch den Staat oder Lebensmitteleinzelhandel) getroffen hat und trifft. Eine intrinsische Motivation und proaktive Eigeninitiative waren bei der Umsetzung gesellschaftlicher Ansprüche bislang nicht vorhanden.

Wie lassen sich die Unternehmen der Fleischbranche also aus diesen festverankerten Handlungsschemata herausholen? - Wann ist der Leidensdruck für ein Umdenken hoch genug? Diese, während der Beschäftigung mit dem Dissertationsthema aufgekommenen Fragen, lassen sich aufgrund der anderweitigen Zielsetzung der vorliegenden Arbeit, nicht final klären. Weitere Fragen, die sich insbesondere für die Praxis mit Blick auf die Zukunft stellen, betreffen darüber hinaus elementare Aspekte hinsichtlich der Umsetzung eines unternehmens- bzw. stufenübergreifenden Reputationsmanagements. Hierfür müssten zunächst die folgenden Ziele definiert werden:

- Wie will man in der Gesellschaft wahrgenommen werden? Welches Reputationsniveau will/kann man unter Kosten-Nutzen-Überlegungen erreichen.

- Wer soll bzw. kann eine derartige Aufgabe erfüllen? Wie soll die Aufgabe institutionell umgesetzt werden? Durch Gremien, QS, Verbände, Leitunternehmen, etc.?

- Welche Ressourcenausstattung will man einer derartigen Institution geben? Welchen Input im Hinblick auf Mitarbeiter, Geld und Managementkapazitäten ist die Branche bereit zu leisten?

- Wie groß ist der Verantwortungsbereich? Horizontale und/oder vertikale Ausrichtung?

- Wie soll die interinstitutionelle Zusammenarbeit zwischen den Unternehmen und der Institution strategisch und operativ ausgestaltet sein und welchen Stellenwert bzw. welche Handlungsbefugnis soll dieser innerhalb der Branche eingeräumt werden?

- Welche Strategie soll bei dem Reputationsaufbau verfolgt werden? Ist es also das Ziel ein aktives Themenmanagement zu betreiben oder durch Schulungen und Hintergrundgespräche Veränderungen zu erreichen? Sollen laute oder leise, mittelbare oder unmittelbare Instrumente zum Einsatz kommen?

Neben dem Input, den die Wissenschaft ganz konkret in den Wandlungsprozess der Fleischwirtschaft geben kann, besteht in verschiedenen Bereichen weiterer Forschungsbedarf für die Agrarökonomie, aber auch für die Wissenschaft insgesamt: 
- Die Fleischwirtschaft wurde im Rahmen der Dissertation als Stellvertreterbranche für den gesamten Agrar- und Ernährungssektor untersucht. Dabei stellt diese einen (negativen) Extrempunkt dar. Daher würde die Analyse der Reputation weiterer Wertschöpfungsketten einen breiteren Überblick über den Status quo des gesamten Agribusiness geben.

- Die in der vorliegenden Dissertation vorgestellten Studien verdeutlichen insbesondere die Notwendigkeit zur Entwicklung eines angepassten Messmodells für die Reputation der Land- und Ernährungsindustrie bzw. von mehrstufigen Branchen insgesamt. Bezogen auf die Agrar- und vor allem die Fleischwirtschaft bietet sich als Anschluss an die vorgestellte Arbeit u. a. insbesondere auf der Stufe der Wurstherstellung und im LEH ein Vergleich der Branchenreputation mit der Reputation eines bekannten Unternehmens an.

- Die präsentierten Beiträge haben gezeigt, dass die grundsätzliche Einstellung zur Fleischwirtschaft sowie das Wissen und der persönliche Bezug in den vorgestellten Studien keinen Einfluss auf das Ansehen der Branche besitzen. Es ist dennoch anzunehmen, dass der Fleischsektor in verschiedenen gesellschaftlichen Gruppen einen unterschiedlichen Ruf genießt. Weiterer Forschungsbedarf besteht somit zum einen, um eine differenziertere Betrachtung des Rufes für verschiedene Gesellschaftsgruppen zu erhalten (z. B. durch eine Clusteranalyse). Zum anderen gilt es, das Ansehen der jeweiligen Stufen der Fleischwirtschaft spezifisch zu analysieren. Bei einer separaten Betrachtung der Stufenreputationen können möglicherweise einzelne funktionale Komponenten einen stärkeren Einfluss auf die Reputation nehmen.

Weiterer grundlegender Forschungsbedarf besteht ferner für das Forschungsfeld der Branchenreputation insgesamt.

- Hierbei könnten eventuelle Effekte einer Divergenz zwischen dem Ruf eines Unternehmens und der Reputation der Branche, in der es arbeitet, untersucht werden. Ist es für ein Unternehmen mit einem guten Ansehen also besser in einer Branche mit einem schlechten Ruf zu arbeiten, oder bringt die entgegengesetzte Konstellation mehr Vorteile (Mahon, 2002)? Die folgenden Aspekte könnten dabei bspw. überprüft werden: 
- Die Reputation eines einzelnen Unternehmens wird durch die Reputation der gesamten Branche beeinflusst/bestimmt.

- Inwieweit beeinflussen sich die verschiedenen Komponenten des Reputationsbegriffs gegenseitig.

- $\quad$ Eine weitere Thematik, die im Rahmen der Branchenreputation diskutiert werden muss, ist das Problem von Free-Rider-Effekten. Insbesondere etablierten Unternehmen eröffnet die Branchenreputation die Möglichkeit den positiven Ruf zu nutzen, ohne sich aktiv am Aufbau und Erhalt der selbigen zu beteiligen (Mahon, 2002). Die folgenden Hypothesen könnten dabei überprüft werden:

- In Industrien mit einer positiven Branchenreputation ist die Gefahr von FreeRidern am größten.

- Nur die Reputation als Ganzes und nicht die einzelnen Komponenten ermöglichen Free-Rider-Effekte.

- Auch bestehen weiterhin im Forschungsfeld der Unternehmens- bzw. Branchenkultur Lücken im Hinblick auf die theoretische Erarbeitung konkreter Umsetzungsstrategien in der Praxis.

Zusammenfassend lässt sich festhalten, dass die vorliegende Dissertation Defizite bezüglich der Reputation der Fleischwirtschaft in der Gesellschaft aufgedeckt hat. Es konnte am Beispiel der Qualitätssicherung gezeigt werden, dass funktionale Maßnahmen zur Verbesserung der Beziehung zur Öffentlichkeit durchaus erfolgreich sind. Dessen ungeachtet besteht für die Systeme jedoch ein latentes Risiko, dass die Gesellschaft bzw. NGOs Schwachstellen enttarnen, die die Glaubwürdigkeit infrage stellen und die Stabilität des Standards gefährden. Schwerpunkt weiterführender Arbeiten muss es demzufolge sein, Konzepte für die Entwicklung und Implementierung eines professionellen Relationship-Managements und von fundierten Kommunikationsstrategien zu generieren, die nicht nur in kritischen Ereignisfällen greifen, sondern bereits proaktiv Krisen vermeiden. 


\section{Literatur}

BMVEL (2005): Zukunft der Nutztierhaltung: Gutachten des Wissenschaftlichen Beirates Agrarpolitik, nachhaltige Landbewirtschaftung und Entwicklung ländlicher Räume beim Bundesministerium für Verbraucherschutz, Ernährung und Landwirtschaft. Unter: URL: http://www.bmelv.de/cae/servlet/contentblob/380386/ publicationFile/21892/Heft508.pdf;jsessionid=289D2EA6A1D4DDBD3C056619 B2F45F92.

Böhm, J., Albersmeier, F., Spiller, A., Zühlsdorf, A. (2009): Zukunftsfaktor gesellschaftliche Akzeptanz: Kommunikation mit der Öffentlichkeit - mehr als KrisenPR. In: Böhm, J., Albersmeier, F., Spiller, A. (Hrsg.) Die Ernährungswirtschaft im Scheinwerferlicht der Öffentlichkeit, Eul-Verlag, 3-16.

Bruhn, M. (2008): Herausforderungen an die Kommunikationspolitik der Unternehmen der Agrar- und Ernährungswirtschaft. In: Spiller, A., Schulze, B. (Hrsg.): Zukunftsperspektiven der Fleischwirtschaft, Universitätsverlag Göttingen, 329-339.

Dyllick, T. (1989): Management von Umweltbeziehungen. Öffentliche Auseinandersetzungen als Herausforderung. Wiesbaden.

Eisenegger, M., Künstle, M. (2003): Reputation und Wirtschaft im Medienzeitalter. In: Die Volkswirtschaft, 58-62.

Engel, P. (2009): Agribusiness als Spielball von Medien und NGOs? In: Böhm, J., Albersmeier, F., Spiller, A. (Hrsg.) Die Ernährungswirtschaft im Scheinwerferlicht der Öffentlichkeit, Eul-Verlag, 19-22.

FAO (2006): Livestock's long shadow - environmental issues and options. Rome.

Gray, E.R., Balmer, J.M.T. (1998): Managing Corporate Image and Corporate Reputation. In: Long Range Planning 31 (5), 695-702.

Gurath, P. (2008): Vom Erzeuger zum Verbraucher - Fleischversorgung in Deutschland. Statistisches Bundesamt. Ausgabe 2008. Wiesbaden.

Herger, N. (2006): Vertrauen und Organisationskommunikation: Identität - Marke Image - Reputation, 1. Aufl., Wiesbaden.

Karmasin, H. (2001): Die geheime Botschaft unserer Speisen. Was Essen über uns aussagt. München.

Mahon, J.F. (2002): Corporate Reputation: Research Agenda Using Strategy and Stakeholder Literature. In: Business \& Society 41 (4), 415-445.

Mai, J. (2009): Argwohn im Anmarsch. In: WirtschaftsWoche vom 26.10.2009. Unter: URL http://www.wiwo.de/karriere/argwohn-im-anmarsch-411743/.

Mast, C., Huck, S., Güller, K. (2005): Kundenkommunikation, Stuttgart.

Schulze, B., Spiller, A. (2008). Trends im Verbraucherverhalten: Ein Forschungsüberblick zum Fleischkonsum. In: Spiller, A., Schulze, B. (Hrsg.): Zukunftsperspektiven der Fleischwirtschaft. Universitätsverlag Göttingen, 233-271.

Schulze, B., Spiller, A., Lemke, D. (2008a): Glücksschwein oder arme Sau? Die Einstellung der Verbraucher zur modernen Nutztierhaltung. In: Spiller, A., Schulze, B. (Hrsg.): Zukunftsperspektiven der Fleischwirtschaft. Universitätsverlag Göttingen, 465-488. 
Schulze, H., Böhm, J., Kleinschmit, D., Spiller, A., Nowak, B. (2008b): Öffentliche Wahrnehmung der Primärverantwortung für Lebensmittelsicherheit: Eine Medienanalyse der Gammelfleischskandale. In: Agrarwirtschaft 57 (7), 334-345.

Spiller, A. (2009): Strategische Spielräume in der deutschen Ernährungsindustrie: Zur Überwindung rigider Branchenkulturen. In: Antoni-Kolmar et al. (Hrsg.) Neue Konzepte der Ökonomik - Unternehmen zwischen Nachhaltigkeit, Kultur und Ethik, Metropolis-Verlag, 349-367.

Weiner, D. (2006): Crisis Communication: Managing corporate reputation in the court of public opinion. In: Ivey Business Journal (März/April), 1-6. 